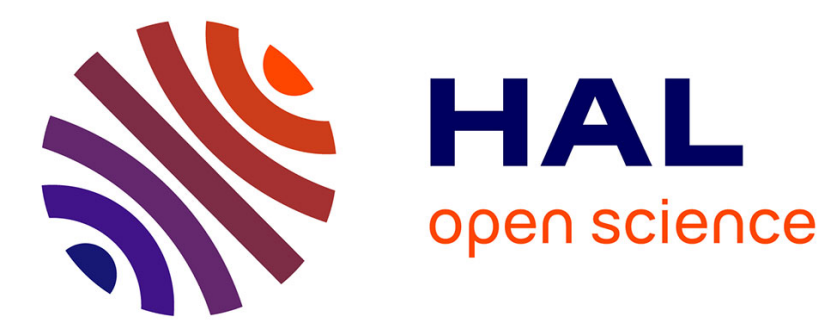

\title{
Les personnes (grammaticales) en relation: ni fusion ni confusion
}

Catherine Filippi-Deswelle

\section{To cite this version:}

Catherine Filippi-Deswelle. Les personnes (grammaticales) en relation: ni fusion ni confusion. Sous la direction de Natalie Depraz. Première, deuxième, troisième personne, ZETA BOOKS, 2014, 978606-8266-86-2. hal-01780736

\section{HAL Id: hal-01780736 \\ https://hal-normandie-univ.archives-ouvertes.fr/hal-01780736}

Submitted on 27 Apr 2018

HAL is a multi-disciplinary open access archive for the deposit and dissemination of scientific research documents, whether they are published or not. The documents may come from teaching and research institutions in France or abroad, or from public or private research centers.
L'archive ouverte pluridisciplinaire HAL, est destinée au dépôt et à la diffusion de documents scientifiques de niveau recherche, publiés ou non, émanant des établissements d'enseignement et de recherche français ou étrangers, des laboratoires publics ou privés. 


\author{
Catherine Filippi-Deswelle \\ (Université de Rouen)
}

\title{
Introduction
}

Cet article, à destination d'un public non spécialiste de linguistique, a pour but de présenter la notion de «personne » dans son acception grammaticale. Il a pour unique ambition de délimiter grossièrement l'inventaire des formes personnelles et d'en dresser l'état des lieux dans le cadre de la théorie linguistique de l'énonciation. Il s'agira de lever un certain nombre de contradictions, voire de confusions, liées à l'emploi du lexème "personne» en grammaire du français et de l'anglais : tout d'abord, je m'interrogerai sur le risque de confusion entre les deux acceptions du terme, à savoir «personne humaine » d'une part, et «personne grammaticale » de l'autre ${ }^{1}$, puis je poserai la question de l'incidence de cette confusion possible sur l'interprétation référentielle du paradigme des pronoms dits «personnels » et j'évoquerai tout particulièrement les travaux de Benveniste à cet égard, et les problèmes posés par leur réception chez les linguistes. Enfin, j'évoquerai le risque de confusion, voire de fusion, entre les personnes lorsqu'il est question de leurs formes plurielles, le pluriel n'étant pas nécessairement indicateur d'addition d'unités de même nature.

\section{Risque de confusion entre "personne grammaticale » et «personne humaine »}

\subsection{Qu'appelle-t-on « personne » en grammaire ?}

L'emploi du métaterme de «personne » (c'est-à-dire dans son acception appliquée au domaine spécialisé de la grammaire) peut prêter à confusion avec l'emploi du terme dans la langue courante pour désigner un être humain ; il en va de même pour l'adjectif «personnel». En effet, lorsque l'on parle des personnes en relation, il importe d'établir s'il s'agit d'un renvoi à des individus, et donc à des référents animés humains de l'univers extralinguistique, ou d'un renvoi aux formes de la langue de type morphèmes disjoints ( $m o i$, toi, lui) ou conjoints (je, $t u, i l$ ), supports de la prédication en accord avec la flexion (ou désinence) verbale correspondante. A cet égard, la mise au point proposée par le linguiste Marc Wilmet est éclairante :

Rappelons par précaution que personnel ne signifie pas « relatif à une personne humaine ou à un animé » (c'est une bévue récurrente, vraisemblablement due au postulat intempestif d'un $i l$ «impersonnel ») mais « exprimant la catégorie de la personne grammaticale ». (Wilmet 2003: 291, $\S 345)$

\footnotetext{
${ }^{1}$ Cet article se propose de prolonger la critique de Claude Hagège à l'égard des thèses de Benveniste sur «le traitement de la personne » au sujet duquel il convient de « distinguer les acceptions sémanticoréférentielle et morphosyntaxique » Hagège 1984: 110. Voir à ce sujet Hagège 1982: 97-98 et plus largement Hagège 1982: 95-100.
} 
Plus précisément, la catégorie ${ }^{2}$ de la personne grammaticale correspond d'une part aux désinences de la conjugaison du verbe (ou flexion verbale), et d'autre part à une classe de mots fermée, à savoir celle des pronoms dits «personnels » ordonnés selon six rangs de personne (première, deuxième et troisième personnes) variables selon le nombre (singulier ; pluriel) et le genre (masculin, féminin, neutre).

En français, on obtient respectivement trois «personnes » au singulier : je, tu, $i l$, elle, et trois «personnes » au pluriel : nous, vous, ils, elles - pour ne citer que les formes en fonction «sujet» du paradigme. Cette catégorie est caractérisée par la notion morphosyntaxique de «variation personnelle» qui prend en compte les paramètres suivants : la fonction et la position syntaxiques, le genre et le nombre. De la position dans la phrase découle la sous-catégorisation suivante en français entre formes conjointes d'une part (qui ne peuvent être séparées du verbe que par l'adverbe de négation ne) et formes disjointes de l'autre (qui manifestent une certaine autonomie) :

formes conjointes + fonction sujet + genre (masc., fém.) + nombre (sg., pl.) : $i l(s) / e l l e(s)$; sujet + nombre seulement (sans le genre) : je, tu, nous, vous ;

formes conjointes + fonction objet direct + genre (masc., fém.) + nombre (sg.) : le, la ; fonction + nombre : me, te, nous, vous; fonction + nombre : les (objet direct) ; leur (objet indirect);

formes disjointes + fonction sujet ou objet ou circonstanciel + genre (masc., fém.) + nombre (sg., pl.) : luileux, ellelelles ; fonction + nombre : moi, toi, nous, vous.

S'y ajoutent les réfléchis (se, soi), les adverbiaux ( $y$ et en) et l'indéfini on (seulement sujet), de même que les possessifs (mon, ton, son, ma, ta, sa, nos, vos, leurs).

En anglais, on obtient en fonction sujet : fonction + nombre sg. I, you ; fonction + genre + nombre sg. he (masc.), she (fém.), it (neutre); l'indéfini one; fonction + nombre pl. we, you, they et en fonction objet (direct et indirect) : fonction + nombre sg. me, you ; fonction + genre + nombre sg. him (masc.), her (fém.), it (neutre); fonction + nombre pl. us, you, them, tandis que my, your, his, her, its (sg.) et our, your, their (pl.) correspondent aux déterminants génitif.

Comme on le voit, plusieurs catégories grammaticales sont mobilisées - le nombre, le genre - lorsque la catégorie de la personne est convoquée, ce qui rend compte de la multiplicité des formes dites personnelles. Par ailleurs, il existe en grammaire des formes dites impersonnelles faisant intervenir la fonction sujet, le nombre singulier et le genre neutre, comme c'est le cas avec $\mathrm{il} / \mathrm{ce} / \mathrm{c}$ ' en français et it en anglais ; on voit que pour $c e / c^{\prime}$ on est également proche de la classe syntaxique des démonstratifs (ce, cet, cette, ces, ceci, cela).

La catégorie grammaticale de la personne présente une homogénéité certaine mais également une hétérogénéité tout aussi évidente. Pour ne citer que les formes «sujet » du paradigme, ce classement indique que le statut de la première et de la deuxième

\footnotetext{
${ }^{2}$ Groussier, Rivière 1996: 32 définissent techniquement le terme de « catégorie » de la façon suivante : «Concept définissant une classe de mots, de marqueurs, de phénomènes, opérations ou fonctions, etc. dans l'analyse linguistique. Ainsi, on parlera de la catégorie de la modalité, de la catégorie du genre, etc. » La catégorie de la personne est une catégorie grammaticale qui se distingue des catégories lexicales. Voir Dubois et al. 1994: 78 ; 355-357 à ce sujet.
} 
personne diffère de celui de la troisième pour ce qui est du marquage du genre et même du nombre dans une certaine mesure : en français, si elle/elles correspond au féminin (sg./pl.), il (sg.) peut renvoyer soit au masculin soit à l'impersonnel selon le contexte d'emploi, tandis que ils n'indique que le masculin pluriel; l'opposition de genre disparaît au profit du masculin lui (objet indirect, $3^{\mathrm{e}}$ pers. sg.), ainsi qu'au pl. avec les (objet direct) et leur (objet indirect), alors que c'est l'opposition de nombre qui n'est plus marquée avec le réfléchi se ( $3^{\mathrm{e}}$ pers.). En anglais, seules les formes de $1^{\mathrm{re}}$ personne (sg. ; pl.), et de $3^{\mathrm{e}}$ personne ( $\mathrm{sg}$. ; pl.) diffèrent tandis que celles de la $2^{\mathrm{e}}$ personne sont invariables. Contrairement au français, la variation en genre est neutralisée à la $3^{\mathrm{e}}$ personne du pl. y compris en fonction sujet et objet (they, them), et c'est it qui est employé pour l'impersonnel. Il importe de préciser que la catégorie du genre en anglais équivaut le plus souvent à une opposition de sexe, contrairement à ce qui se passe en français où le genre est davantage grammaticalisé et indépendant de considérations extralinguistiques faisant référence à l'univers physico-culturel.

Je reviendrai ultérieurement sur d'autres distinctions fondamentales entre $1^{\text {re }}$ et $2^{\mathrm{e}}$ personnes d'une part et la $3^{\mathrm{e}}$ personne de l'autre. Pour l'heure, le fait que la distinction de genre s'appuie à des degrés divers d'une langue à l'autre sur l'opposition de sexe (ou genre naturel en grammaire) me conduit à nouveau à m'interroger sur le risque de confusion entre les diverses acceptions du mot « personne » et sur les raisons sous-tendant une telle confusion. Wilmet (2003: 291) évoquait l'opposition «personnel/impersonnel» comme origine possible de cette dernière; l'histoire de l'étymologie du terme et de ses dérivations sémantiques successives est, pour sa part, également éclairante.

\subsection{Evolution sémantique et traductions problématiques du lexème «personne »}

\subsubsection{Etymologie du mot "personne»: de l'étrusque au latin, et du latin au} français

Le linguiste Emile Benveniste évoque les diverses origines du mot «personne », et ce plus particulièrement au sujet de la flexion verbale :

Dans toutes les langues qui possèdent un verbe, on classe les formes de la conjugaison d'après leur référence à la personne, l'énumération des personnes constituant proprement la conjugaison ; et on en distingue trois, au singulier, au pluriel, et éventuellement au duel. Cette classification est notoirement héritée de la grammaire grecque, où les formes verbales fléchies constituent des $\pi \rho o ́ \sigma \omega \pi \alpha$, des personae, des «figurations » sous lesquelles se réalise la notion verbale. (Benveniste 1966: 225)

Or, comme le titre de l'article de Jack Schmidely l'indique - «Controverse à propos du système de la personne » (1976) - rien n'est établi une fois pour toutes chez les linguistes lorsque l'on traite de la catégorie de la personne grammaticale. Il est ici question de répondre à un article d'André Joly, "Sur le système de la personne » (1973), qui critique les vues d'Emile Benveniste (1966), notamment au sujet de l'étymologie du mot « personne»: 
En latin, «le terme de grammaire apparaît au moment où persona signifie "masque", "personnage d'une pièce" (avec une valeur fonctionnelle), "rôle théâtral", ... » (Joly, p. 45); n'est-ce pas là confirmer l'application du terme par référence aux seuls vrais «acteurs » de l'acte de langage, à ceux qui parlent effectivement: les différents protagonistes du procès de l'énonciation? Ce n'est que par la suite que de «rôle théâtral »s'effectuera le passage à tout "individu ", l'extension étant déjà perceptible et même de façon plus large dans le domaine grammatical. La concordance entre l'emploi théâtral et l'emploi grammatical de persona me semble plutôt être un argument en faveur de la thèse de Benveniste que le contraire! (Schmidely 1976: 61)

Avant de développer cette dernière, il convient de s'attarder sur les acceptions « masque / rôle de théâtre ${ }^{3}$ » dans la mesure où il y aurait à l'origine du mot latin un emprunt à l'étrusque $\mathrm{fersu}^{4}$ qui selon Wilmet a signifié "'porte-voix', puis 'acteur masqué' » (2003: 290).

Comme Joly (1973) et Schmidely (1976) qui établissent un lien entre théâtre et grammaire, François Jacquesson (2008: 22) précise qu'un autre lexème, imagines, était également usité dans ce sens, en concurrence avec persona («masque (des ancêtres) »). Il explique que le «choix d'un vocabulaire technique » est lié à « un contexte culturel plus vaste que la grammaire » $(2008: 35)$ et que le mot persona en est venu à se spécialiser dans le domaine du théâtre avec pour sens « rôle » :

Il semble qu'en étrusque le mot était phersu, mais les Latins ne connaissaient pas le son «aspiré »ph (ils ont réduit le mot grec amphora à la forme ampulla d'où le français ampoule), et le mot s'est latinisé en personne. Du sens de «masque » chez les Latins et peut-être chez les Etrusques déjà, on est passé au sens de «rôle », au théâtre. Mais déjà Cicéron employait le mot au sens qui est courant chez nous, de «personne », «individu humain ». (Jacquesson $2008: 22$ )

Et de là, en tant que synonyme de «fonction, posture, figure », il a pris celui de «personne grammaticale». Françoise Létoublon (1994: 9) note qu'en latin, l'évolution sémantique de persona est attestée : «masque de l'acteur > personnage joué par cet acteur > rôle grammatical (joué par les «actants-acteurs » d'une phrase ; unité linguistique, phrase ou discours, vue comme un «drame » ou une «scène ») ». Mais elle montre également que le terme latin-étrusque persona («masque ») pour renvoyer à la notion de personne grammaticale, et donc à un usage technique du

\footnotetext{
${ }^{3}$ Voir la définition du Dictionnaire de la langue française, Paris, Le Petit Robert, 1990, p. 1409 :

«Personne n.f. (1180 ; lat. persona "personnage, personne", mot d'o. étrusque "masque de théâtre").

1) Individu de l'espèce humaine $[. .$.

5) Gram. (h. XIII $I^{e}$; persone, $X V^{e}$ ) "Indication du rôle que tient celui qui est en cause dans l'énoncé, suivant qu'il parle en son nom (première personne), qu'on s'adresse à lui (deuxième personne) ou qu'on parle de lui (troisième personne)" (MAROuZEAu). Première (V. Je, nous), deuxième (V. Tu, vous), troisième (V. Il, elle) personne d'un verbe. Roman écrit à la première, à la troisième personne. - Par anal. Psychologie à la première personne (subjective), à la troisième personne (objective). "Toute métaphysique est à la première personne du singulier. Toute poésie aussi." (ARAGON). »

${ }^{4}$ Aussi orthographié phersu, comme dans le Dictionnaire historique de la langue française, dans lequel il est fait état de l'évolution suivante du mot latin persona: «les grammairiens, depuis Varron, l'utilisent pour traduire le grec prosôpon [...]. Le mot serait un emprunt technique à l'étrusque phersu qui, d'après l'inscription où se lit le mot, désigne un masque ; cependant, le rapport entre l'étrusque et le grec est difficile à déterminer » (1998, F-PR : 2677). Voir ci-après Létoublon 1994 à ce sujet.
} 
terme, vient de la traduction du mot grec $\pi \rho o ́ \sigma \omega \pi o v$ qui désignait le «vis-à-vis » et signifiait «visage » ou «façade » ${ }^{5}(1994: 8)$. Ce qui la conduit à remettre en cause l'évolution sémantique précédemment évoquée: «masque / masque de théâtre $>$ personne grammaticale ».

\subsubsection{Nécessité de remonter au grec pour mieux comprendre le rapport latin- français}

Ainsi, il convient de remonter au grec pour mieux comprendre le rapport latinfrançais :

La notion de personne, objet de nos débats, nous vient en tant que terme grammatical technique des grammairiens grecs [...]. Mais les grammairiens grecs n'ont pas construit leur terminologie à partir de rien : je voudrais pour ma part examiner brièvement, à partir de l'examen des emplois du terme qu'ils ont adopté comme terme technique, $\pi \rho o ́ \sigma \omega \pi o v$, comment la personne grammaticale se dégage progressivement en grec de la notion essentielle de «visage », «figure humaine»: en somme, ce sont les soubassements anthropologiques de la notion dans les mentalités, préalable nécessaire à l'élaboration d'une terminologie grammaticale, qui m'intéresseront.

La filiation latine de persona, «masque de théâtre » à persona > personne au sens de «personne grammaticale» me semble avoir produit sur le public, même chez les linguistes, un effet pervers, ou même une illusion : on croit souvent que la notion de personne grammaticale provient directement du nom du masque et de l'expérience du théâtre. J'essaierai de montrer que la «personne » en grammaire, avant d'être liée au personnage de théâtre, se rattache à l'expérience collective du face à face avec autrui, à la rencontre de l'autre et la découverte en lui d'un autre soi-même. En effet, avant la persona des grammairiens romains, les grammairiens grecs utilisent le terme $\pi \rho o ́ \sigma \omega \pi o v$, que les grammairiens latins ont transcrit par le terme cité. (Létoublon $1994: 7-8$ )

Létoublon évoque donc un problème de traduction du grec vers le latin et récuse l'hypothèse selon laquelle les deux termes auraient connu la même filiation sémantique :

En utilisant le nom du masque de théâtre pour exprimer la notion grammaticale de personne, les grammairiens latins ont certainement cru traduire l'usage des grammairiens grecs puisque le grec $\pi \rho o ́ \sigma \omega \pi o v$ qui désigne chez eux la personne grammaticale se rencontre aussi à la même époque en grec dans le sens de «masque de théâtre ». Mais cela n'implique pas pour autant que la filiation ait été la même dans les faits en grec qu'en latin. [...] En héritant de l'ambiguïté initiale du mot avec déplacement (grec visage/personne/masque, latin masque/personne) le français a oublié le sens de masque. (Létoublon 1994 : 9-10)

\footnotetext{
${ }^{5}$ L'étymologie du mot grec permet la définition suivante de $\pi \rho o ́ \sigma \omega \pi o v$ d'après Létoublon 1994: 9 : préposition $\pi \rho o ́ \varsigma$ «devant»+ ancien nom de l'œil = «ce que l'on a devant les yeux », «le «vis-àvis », qu'il s'agisse d'un être humain ou d'un monument. »
} 
La conclusion de ces analyses, selon cette linguiste, oriente sans équivoque l'interprétation vers un lien originel avec la personne humaine : «Il semble bien que dans les cultures anciennes, la terminologie de la personne repose sur la notion de «personne humaine », soit comme être humain caractérisé par un visage en grec, soit comme analogue d'un personnage de théâtre en latin » (1994:11).

A l'issue de cette étude étymologique de la notion de "personne », il apparaît que le risque de confusion entre «personne humaine » et «personne grammaticale » est fondé ; il repose sur des considérations historiques, anthropologiques et culturelles. Chez les linguistes, le problème se pose depuis l'Antiquité et ne semble pas être résolu uniformément. On l'a vu, alors qu'avec l'étymologie du terme latin-étrusque persona, on croyait pouvoir se passer (relativement) de l'analogie avec la personne humaine pour privilégier une acception plus fonctionnelle et abstraite de représentant d'un rôle syntaxique dans la phrase, avec celle du terme grec $\pi \rho o ́ \sigma \omega \pi o v$, une telle analogie reprend tous ses droits, comme antérieure à la dérivation sémantique du mot vers son usage technique en grammaire.

Mais dans quelle mesure est-il pertinent de chercher à dé-personnaliser, pour ainsi dire, la catégorie grammaticale de la personne, comme le préconise Wilmet (2003 : 291)?

\section{Les personnes en relation... d'opposition (sans fusion, ni confusion)}

Je vais à présent aborder la question de l'incidence de cette confusion possible entre «personne humaine» et «personne grammaticale» sur l'interprétation référentielle du paradigme des pronoms dits «personnels», et j'évoquerai tout particulièrement les travaux de Benveniste à cet égard, et les problèmes posés par leur réception chez les linguistes.

Afin de dé-fusionner nos deux ordres de problèmes pourtant intrinsèquement liés la référence à l'être humain et l'emploi grammatical - il convient de partir du contexte linguistico-culturel à l'origine de la notion de personne grammaticale, pour établir le fait qu'ils entrent en relation d'analogie par dérivation sémantique ordonnée de manière non aléatoire, et en relation de contiguïté de type métonymique, dans la mesure où d'une part c'est la personne humaine qui, le cas échéant, utilisait un portevoix ou portait un masque de théâtre, et où d'autre part le masque était là pour signifier deux choses : la première, qu'il s'agissait d'un être humain faisant fonction d'acteur, et la seconde, que l'acteur jouait le rôle d'un personnage. Ce dernier terme renvoie à une posture, à une «figuration» (Benveniste 1966: 225), d'ordre symbolique et signifiant, qui est directement ancrée sur la situation d'énonciation dans laquelle les protagonistes du discours prennent tour à tour la parole. L'inconvénient majeur de cette analogie entre «personne humaine » et «personne grammaticale » est qu'elle semble imposer au terme de «pronom personnel » non seulement une interprétation en termes de genre naturel, où les emplois «personnels » s'opposent à l'emploi dit «impersonnel », mais aussi une lecture homogène des emplois «personnels », dès lors indifférenciés les uns des autres, et sujets à la confusion, voire à la fusion, référentielle.

En effet, les pronoms personnels ont pour certains un rôle proprement énonciatif et pas seulement prédicatif (ou syntaxique) ${ }^{6}$. Il est donc nécessaire de mettre au jour leur

\footnotetext{
${ }^{6}$ Lorsqu'ils sont employés en fonction «sujet» dans l'énoncé, les pronoms personnels ont une fonction prédicative en tant que support de la prédication, à savoir la mise en relation syntaxique du
} 
fonctionnement en contexte, dans le cadre d'une configuration bien délimitée pour chacun d'eux : les personnes (grammaticales) sont certes en relation, mais surtout en relation d'opposition. En fait, il apparaît assez nettement que les personnes grammaticales forment un système linguistique qui s'organise selon différents microsystèmes: elles sont donc nécessairement en relation, dans la mesure où, conformément à la définition même de "système », chacune tire ses caractéristiques propres de celles des autres dans une relation de différenciation - ce qui élimine les risques de confusion. Cependant, la dénomination unifiée de «pronoms personnels » fait problème, en ce qu'elle masque précisément le micro-système entre $1^{\mathrm{re}}$ et $2^{\mathrm{e}}$ personnes et la $3^{\mathrm{e}}$ personne d'une part, et entre la $1^{\text {re }}$ et la $2^{\mathrm{e}}$ personne de l'autre. Benveniste ne dit pas autre chose à propos de la flexion verbale :

Ces dénominations ne nous renseignent ni sur la nécessité de la catégorie, ni sur le contenu qu'elle implique ni sur les relations qui assemblent les différentes personnes. Il faut donc rechercher comment chaque personne s'oppose à l'ensemble des autres et sur quel principe est fondée leur opposition, puisque nous ne pouvons les atteindre que par ce qui les différencie. (Benveniste $1966: 226$; c'est moi qui souligne)

D’après Jacquesson, ces différents micro-systèmes - qui ont été théorisés par Benveniste (1966: 231-232) en termes de «corrélation de personnalité » et «corrélation de subjectivité » - s'infèrent de la nature même de la situation d'énonciation qui reproduit la situation de communication des acteurs de théâtre :

Il n'est pas indifférent de constater que la stylisation propre au théâtre antique romain, où les personnages valent moins pour eux-mêmes que, dans les dialogues de scène, pour le relief qu'ils se donnent l'un à l'autre en s'opposant, sont une première théorie moderne de la personne. Car pour les linguistes antiques déjà, il y a trois personnes comme dans une situation de théâtre : celui qui parle (moi), celui à qui je parle (toi), et celui dont toi et moi parlons (lui). La mise en scène du dialogue, et la prise de conscience de la technique des répliques chez les auteurs comme chez les scénaristes n'est sans doute pas étrangère à l'individuation sous le nom de personne (masque) de ces situations éternellement récurrentes de celui qui parle, celui qui écoute, et le sujet de leur conversation - qui peut-être va bientôt sortir des coulisses, son bâton à la main, ou avec son air fat. (Jacquesson $2008: 22$ )

Or, outre l'emploi de « personne / personnel », un autre métaterme contribue ici au brouillage des relations: celui de «pronom». Il convient donc également de s'attarder sur son sémantisme avant de présenter l'apport de Benveniste quant au fonctionnement de la catégorie de la personne grammaticale.

\subsection{Origine et définition du métaterme « pronom »}

Considérons pour commencer l'origine et la définition du métaterme " pronom » avec Jacquesson (2008: 28) qui cite «le premier manuel de grammaire écrit en Occident, la Technè Grammatikè attribuée à Denys le Thrace (fin du II ${ }^{\mathrm{e}}$ siècle AEC) » (2008: 20) :

sujet et du prédicat (entendu ici au sens large comme renvoyant au verbe et à ses compléments). Ils désignent donc l'entité dont on parle. 
La $[\ldots]$ grammaire a un chapitre sur les pronoms (antônumia ${ }^{11}$ ) et dit (Lallot $1998: 61)$ :

«Le pronom est un mot employé en place d'un nom, et qui indique des personnes définies. »

$<$ La personne (prosôpon), avec le genre, le nombre et le cas, est l'une de ses propriétés («accidents »)>

Note 11 : C'est-à-dire anti-onuma, avec anti «à la place de »; les Latins traduiront par pro-nomen, qui est un calque. (Jacquesson 2008: 28 ; mes précisions entre crochets)

La question qui se pose alors concerne la construction de la référence du pronom ainsi mis en relation avec la catégorie lexicale du nom, avec laquelle il entretient un rapport de substitution notionnelle, sémantique, et de cohésion textuelle, notamment sur le plan de l'antécédence syntaxique (accord en genre et en nombre). La catégorie grammaticale du pronom convoque donc la catégorie sémantique de l'anaphore. En va-t-il ainsi à toutes les personnes ? Rien n'est moins sûr pour les linguistes. Citons encore Jacquesson (2008: 83) au sujet de l'exemple (1) :

(1) Jules est un sale type. Je ne peux pas le sentir.

On entend ordinairement par pronom un élément de la chaîne parlée (mot indépendant ou non [...]) qui a même contenu sémantique qu'un autre élément à quoi il se substitue. Si je dis Jules est un sale type. Je ne peux pas le sentir, le le du second énoncé a toute chance de représenter Jules, quelle que soit la ponctuation présumée ou le type de pause entre les deux énoncés, que le soit enclitique ou suffixé ou indépendamment constitué. Les pronoms de ce type, qui sont des «pronoms vrais » [...], n'ont aucun contenu par euxmêmes, et ne fonctionnent qu'autant qu'il existe un contenu latent. Ce contenu peut avoir été donné dans les énoncés précédents, ou être évidents aux interlocuteurs. (Jacquesson $2008: 83$ )

L'anaphore peut en effet être de type contextuel (ou endophorique) ou situationnel (ou exophorique). Il apparaît que ce qui convient pour la $3^{\mathrm{e}}$ personne (sg.; pl.) ne fonctionne plus aux $1^{\text {re }}$ et $2^{\mathrm{e}}$ personnes. En effet, il est impossible de leur associer un nom du lexique lors de l'identification de la référence de je ou $t u$, à moins qu'il ne s'agisse de noms propres (prénom, nom de famille, titre), mais alors la substitution est agrammaticale en l'état, comme (2) l'indique :

(2) Jules est un sale type. Je /* François / * Catherine ne peux pas le sentir.

Seul le rétablissement de l'accord entre le sujet et le verbe peut rendre l'énoncé acceptable :

(3) Jules est un sale type. François $(=\mathrm{il} / *$ je $) /$ Catherine $(=$ elle $/ *$ je $)$ ne peut pas le sentir.

Le problème est que (2) et (3) ne sont absolument pas interchangeables à signification constante. Même si je est identifiable à François ou à Catherine, il reste 
que s'ils souhaitent prendre la parole ici et maintenant en leur nom, ils sont contraints de recourir à je uniquement.

Il en va de même à la $2^{\mathrm{e}}$ personne du sg., où $t u$ est identifiable à Natalie ou à Philippe :

(4) Qu'en penses-tu ?

(5) *Qu'en penses Natalie / Philippe ?

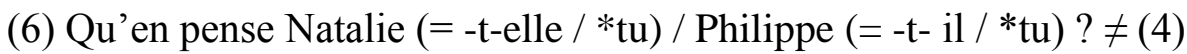

Ainsi, il apparaît très clairement que les $1^{\mathrm{re}}$ et $2^{\mathrm{e}}$ personnes $\mathrm{du} \mathrm{sg}$. forment un micro-système par opposition à la $3^{\mathrm{e}}$. Précisons que le cas du pluriel sera évoqué ultérieurement, mais que le raisonnement s'applique aussi à la $3^{\mathrm{e}}$ pers. du pl. Seuls les pronoms de $3^{\mathrm{e}}$ personne sont des pronoms au sens grammatical du terme, à savoir entrent dans une relation anaphorique avec un antécédent nominal. En revanche, les $1^{\text {re }}$ et $2^{\mathrm{e}}$ personnes sont des «personnes » au sens délimité plus haut de «rôle» exclusivement énonciatif. Ce fait est établi depuis l'Antiquité gréco-latine, mais il ne transparaît pas clairement dans la terminologie grammaticale qui en a découlé puisque les termes de «personne » et de «pronom»s'appliquent indifféremment aux six rangs de personne (sg. et pl.), au sein de la sous-classe des «pronoms » appelés «personnels». En citant Denys le Thrace, Jacquesson explique comment ce glissement terminologique à valeur globalisante s'est effectué :

La Technè grammatikè [...] distribuait les personnes dans une sorte de géographie fonctionnelle ${ }^{2}$ :

«Les personnes (prosôpon) sont trois : première, deuxième, troisième. La première, c'est de qui [vient] la parole (aph'hou ho logos), la deuxième c'est vers qui [va] la parole (pros hon ho logos), la troisième c'est autour de qui [tourne] la parole (peri hou ho logos). »

Note 2: La Grammaire de Denys le Thrace, traduite et annotée par Jean Lallot 1998 : 56. Lallot traduit la phrase grecque ainsi : «La première, c'est celui de qui vient l'énoncé, la deuxième, celui à qui il est adressé, la troisième, celui dont il parle. » (Jacquesson $2008: 20$ )

\subsection{Les pronoms « personnels » ne sont pas des pronoms}

A la suite des travaux de Benveniste (notamment «Structure des relations de personne dans le verbe ») qui ont remis en cause cet héritage classique à la lumière de la morphologie des verbes de langues arabes et sémitiques et de leurs grammaires,

Une théorie linguistique de la personne verbale ne peut se constituer que sur la base des oppositions qui différencient les personnes; et elle se résumera toute entière dans la structure de ces oppositions. Pour les déceler, on pourra partir des définitions que les grammairiens arabes emploient. Pour eux, la première personne est al-mutakallimu, « celui qui parle »; la deuxième almuhatabu, «celui à qui on s'adresse »; mais la troisième est al-ya'ibu, «celui qui est absent». Dans ces dénominations se trouve impliquée une 
notion juste des rapports entre les personnes; juste surtout en ce qu'elle révèle la disparité entre la $3^{\mathrm{e}}$ personne et les deux premières. Contrairement à ce que notre terminologie ferait croire, elles ne sont pas homogènes. C'est ce qu'il faut d'abord mettre en lumière. (Benveniste 1966 : 227-228; c'est moi qui souligne)

Jacquesson, après d'autres ${ }^{7}$, a établi l'opposition entre «pronoms vrais » et «personnes vraies » sur les bases de manipulations de type (1)-(6), comme celles que j'ai effectuées précédemment à partir de son exemple (1) :

Les pronoms personnels ne se substituent pas à un nom. C'est pourquoi dans beaucoup de langues les «pronoms de $3^{\mathrm{e}}$ personne »- qui eux peuvent se substituer à des noms - sont en réalité des démonstratifs transformés en pronoms. Ce sont, parmi les pronoms dits personnels, les seuls pronoms vrais et souvent les seuls à suivre les catégories nominales comme le genre.

Mais les pronoms de $1^{\text {re }}$ et $2^{\mathrm{e}}$ personne ne se substituent aucunement à des noms explicités par des énoncés antérieurs ou implicites aux interlocuteurs. Ils n'identifient pas quelque chose puisque «moi » et «toi », les acteurs du dialogue, sont nécessairement des animés humains. Mais ils n'identifient pas non plus quelqu'un, puisque «je " change d'identification à chaque fois qu'un autre prend la parole. Ils ne sont nullement le résultat d'une thématisation antérieure. Bien au contraire, les pronoms personnels, dont l'assignation change à chaque nouvel acte de parole, ne désignent rien d'autre que les positions relatives dans cet acte de parole : «moi » est celui qui parle, «toi » celui à qui «je» parle. Les pronoms personnels ne sont donc pas des pronoms. [...]

A certains égards, les mots qui désignent les vraies personnes (la $1^{\mathrm{re}}$ et la $2^{\mathrm{e}}$ ), et qui ne sont pas des pronoms vrais, sont plus proches des déictiques ${ }^{8}$ dont le système $[\ldots]$ est souvent organisé en fonction du locuteur, à partir duquel l'espace de repérage est structuré. [...]

On pourrait voir dans le système des personnes vraies une sorte de deixis spécialisée, et réduite au domaine très spécifique des fonctions relatives à l'acte de parole. (Jacquesson $2008: 88-90$ )

\footnotetext{
${ }^{7}$ Voir Maingueneau 1990: 6 au sujet du « couple je-tu, véritables «personnes » du dialogue » à côté du « pronom il, véritable pro-nom, que Benveniste préfère placer dans le registre de la non-personne, celui des objets du monde autres que les interlocuteurs ». Et aussi Maingueneau 1999: 23-24 sur les différences entre les «personnes » et la «non-personne ».

${ }^{8}$ Voir Jakobson 1963: 178 qui rassemble en une seule catégorie les pronoms personnels et les déictiques, dénommés embrayeurs dans la traduction française du terme anglais shifters : «Tout code linguistique contient une classe spéciale d'unités grammaticales qu'on peut appeler les embrayeurs ${ }^{3}$ : la signification générale d'un embrayeur ne peut être définie en dehors d'une référence au message »; note 3 : «NDT : nous avons choisi ce terme pour traduire l'anglais Shifter, emprunté par Jakobson à O. Jespersen, Language, pp. 123-124. Jespersen définit ainsi le shifter : "une classe de mot... dont le sens varie avec la situation... exemple papa, maman, etc..." Le mot "embrayeur", qui est utilisé dans le langage technique pour traduire certains des sens de shift, shifter, nous a paru propre à désigner ces unités du code qui "embrayent" le message sur la situation. » Les déictiques en français sont ici, là, làbas, maintenant et alors, ceci et cela, celui-ci et celui-là, voici et voilà ; en anglais here et there ; now et then; this et that.
} 
Il s'agit là de la conséquence de la «corrélation de personnalité » qui, selon Benveniste, oppose « les personnes je/tu à la non-personne $i l »(1966: 235)$. En effet, si les «personnes» (ou «personnes vraies» dans la terminologie de Jacquesson) s'opposent à la «non-personne» (ou «pronoms vrais»), alors j'ajouterai que les «pronoms » (ou «pronoms vrais ») s'oppose aux «non-pronoms » (ou «personnes vraies »), au risque de m'exposer à une réception aussi houleuse que celle qu'a connu la «non-personne » de Benveniste, lors de l'analyse faite par ce linguiste du système de la personne chez les grammairiens arabes. Creissels (1995), notamment, fait remarquer de manière critique que :

Les pronoms dits de première et de deuxième personne ont nécessairement pour référent une personne humaine. [...] La «troisième personne » des grammairiens quant à elle ne se limite pas aux personnes humaines. Mais elle ne les exclut pas non plus. De ce fait, substituer «non-personne» à «troisième personne », comme le préconisait Benveniste, ne fait qu'accroître la confusion, d'autant plus que «non-personne » évoque irrésistiblement « impersonnel ». (Creissels $1995: 121)$

\subsection{Fonction énonciative et fonction prédicative}

Moignet (1972) et Joly (1973 [1987] ; 1994) ont également fortement critiqué cette approche dans le cadre théorique énonciatif de la Psychomécanique du langage, élaboré par le linguiste Gustave Guillaume, sur la base de la prééminence de la $3^{\mathrm{e}}$ personne sur les $1^{\mathrm{re}}$ et $2^{\mathrm{e}}:$ je/tu ont pour référent un nom propre qui s'accorde à la $3^{\mathrm{e}}$ personne (comme vu dans les exemples (3) et (6) ci-dessus). Joly (1994: 48 ; ses italiques) précise ainsi que «sous toute personne, il y a une troisième personne » et que l'analyse de Benveniste sur la non-personne « repose, dans le meilleur des cas sur un malentendu, dans le pire, sur un contresens quant à la nature de la personne dans le langage » (Joly [1973] 1987). Il me semble qu'on ne peut guère faire cette critique à Benveniste qui présente les deux fonctions, énonciative et prédicative, ensemble - il réfère en particulier à la fonction prédicative en recourant à la notion de « discours / indication d'énoncé sur »-, pour ensuite établir la « corrélation de personnalité »:

Dans les deux premières personnes, il y a à la fois une personne impliquée et un discours sur cette personne. «Je » désigne celui qui parle et implique en même temps un énoncé sur le compte de «je »: disant «je », je ne puis pas ne pas parler de moi. A la $2^{\mathrm{e}}$ personne, «tu » est nécessairement désigné par « je » et ne peut être pensé en dehors d'une situation posée à partir de « je »; et, en même temps, "je » énonce quelque chose comme prédicat de " tu». Mais à la $3^{e}$ personne, un prédicat est bien énoncé, seulement hors du «je$t u »$; cette forme est ainsi exceptée de la relation par laquelle «je » et «tu» se spécifient. Dès lors la légitimité de cette forme comme «personne» se trouve mise en question. Nous sommes ici au centre du problème. La forme dite de $3^{e}$ personne comporte bien une indication d'énoncé sur quelqu'un ou quelque chose, mais non rapporté à une «personne » spécifique. [...]

Il s'ensuit que, très généralement, la personne n'est propre qu'aux positions «je » et «tu ». La $3^{\mathrm{e}}$ personne est, en vertu de sa structure même, la forme non-personnelle de la flexion verbale. (Benveniste 1966 : 228 ; 230 ; c'est moi qui souligne) 
Il est vrai, cependant, que les propos de Benveniste peuvent prêter à confusion lorsqu'il est précisé quelques pages plus loin qu'on doit «prendre pleinement de cette particularité que la «troisième personne » est la seule par laquelle une chose est prédiquée verbalement » (1966: 230). Il s'agirait d'une contradiction s'il était alors question de restreindre la fonction prédicative à la $3^{\mathrm{e}}$ personne. On peut néanmoins comprendre le mot «chose» comme référant à une entité inanimée sur le plan sémantique, et dans ce cas on éviterait l'incompréhension. Pour ma part, je m'en tiendrai à la p. 228 qui me semble claire (cf. citations précédente et suivante) :

Nous sommes ici au centre du problème. La forme dite de $3^{\mathrm{e}}$ personne comporte bien une indication d'énoncé sur quelqu'un ou quelque chose, mais non rapporté à une "personne» spécifique. L'élément variable et proprement "personnel » de ces dénominations fait ici défaut. C'est bien l'« absent » des grammairiens arabes. Il ne présente que l'invariant inhérent à toute forme d'une conjugaison. La conséquence doit être formulée nettement : la $3^{\mathrm{e}}$ personne n'est pas une «personne »; c'est même la forme verbale qui a pour fonction d'exprimer la non-personne. (Benveniste 1966 : 228 ; c'est moi qui souligne)

Seules les $1^{\text {re }}$ et $2^{\mathrm{e}}$ personnes (les «personnes vraies ») cumulent les deux fonctions, énonciative ( $d$ ' « embrayage ${ }^{9}$ sur la situation d'énonciation quant à leur identification référentielle) et prédicative (en tant que terme de départ de l'énoncé), tandis que la $3^{\mathrm{e}}$ personne a seulement une fonction prédicative (de type anaphorique avec un «pronom vrai »). Il convient d'observer à cet égard que c'est sans doute ce qui fonde l'apparente homogénéité du paradigme des pronoms dits personnels : leur propriété d'être support de la prédication - qu'ils relèvent de la «personne » ou de la «non-personne »- et, le cas échéant, support du marquage de l'accord entre le sujet et le prédicat verbal ${ }^{10}$.

Schmidely (1976 : 57-63) a, pour sa part, réfuté les critiques de Joly (1973) en soulignant que :

Pour retrouver le fondement des distinctions personnelles il faut tenter de voir en quoi s'opposent les entités-supports concernées : ou bien il s'agit des deux entités directement liées au fonctionnement de la communication - le locuteur et son allocutaire - ou bien il ne s'agit ni de l'un ni de l'autre, caractérisation doublement négative (Joly, p. 14, le rappelle fort justement) qui justifie encore l'appellation suggestive de non-personne. [...] Benveniste n'a jamais exclu la non-personne du système inter-personnel. Au contraire,

\footnotetext{
${ }^{9}$ Voir l'expression «l'élément variable » dans la citation de Benveniste 1966: 228 qui évoque le rôle de shifter propre aux «personnes (vraies)». Dans «De la subjectivité dans le langage», Benveniste précise que je est « un terme qui ne peut être identifié que dans ce que nous avons appelé ailleurs [voir Benveniste 1966: 252-255] une instance de discours et qui n'a de référence qu'actuelle. La réalité à laquelle il renvoie est la réalité du discours » (Benveniste 1966: 261-262). Il ajoute que « le langage propose en quelque sorte des formes "vides" que chaque locuteur en exercice de discours s'approprie et qu'il rapporte à sa "personne", définissant en même temps lui-même comme je et un partenaire comme $t u$. L'instance de discours est ainsi constitutive de toutes les coordonnées qui définissent le sujet » (Benveniste 1966: 263), à savoir le couple « je-tu » en association avec les déictiques.

${ }^{10}$ Wilmet 2003: 293, § 346 fait remarquer à cet égard que «les grammaires identifient la première personne avec la "personne qui parle", la deuxième personne avec la "personne à qui on parle" et la troisième personne avec la "personne de qui on parle". En réalité, la première personne et la deuxième personne sont l'une et l'autre des "personnes de qui on parle", mais la première personne parle ellemême d'elle-même et la deuxième personne est celle à qui on parle d'elle-même. »
} 
toute son entreprise a été de définir la place exacte que celle-ci occupe dans la catégorie et de dégager les deux corrélations complémentaires qui président à l'organisation du système : corrélation de personnalité - où « la troisième personne $»^{11}$ représente en fait le membre non-marqué » (souligné par nous, Benveniste 1956, in 1966b, p. 255) - et corrélation de subjectivité. (Schmidely $1976: 62$ )

\subsection{Ambiguïté de l’opposition « personne/non-personne »}

Le métaterme forgé par Benveniste prête éventuellement à confusion pour les raisons évoquées précédemment au sujet de l'origine du mot «personne » et de son lien avec la personne humaine, nécessairement sexuée : d'un côté, la $3^{\mathrm{e}}$ personne étant la seule à indiquer le genre et le trait /animé/ à côté du trait/non-animé/, il semble impropre de la désigner comme «non-personne»; de l'autre, on sait que le genre s'est grammaticalisé et que la $3^{\mathrm{e}}$ personne peut référer à de l'inanimé (masc. ; fém.), et dans ce cas le terme de «non-personne » fait sens. Benveniste lui-même fait un usage trop peu explicité des guillemets et des italiques : il évoque la «personne ${ }^{12}$ (1966 : 263) du locuteur, entendu comme «l'homme qui parle» (1966:266), ce qui désigne donc le référent humain de l'instance de discours, tandis que précédemment (1966 : 260), en définissant le sujet parlant comme « ego »- «Est « ego » qui dit « ego ». »-, il avançait que «nous trouvons là le fondement de la « subjectivité », qui se détermine par le statut linguistique de la «personne », en précisant que c'est la «condition de dialogue qui est constitutive de la personne», mis cette fois en italiques. Dans ce cas, il opère un glissement vers sa propre acception du terme «personne»dans le cadre de la catégorie grammaticale de la personne. Mais l'ambivalence demeure lorsque le mot est employé en caractères droits sans être mis entre guillemets $^{13}$ : Benveniste précise que «l'installation de la «subjectivité » dans le langage crée, dans le langage, et croyons-nous hors du langage aussi bien, la catégorie de la personne » $(1966: 263)$. Il dit encore :

Ainsi, en toute langue et à tout moment, celui qui parle s'approprie je, ce je qui, dans l'inventaire des formes de la langue, n'est qu'une donnée lexicale pareille à une autre, mais qui, mis en action dans le discours, y introduit la présence de la personne sans laquelle il n'est pas de langage possible. [...] Que l'un des hommes les prononce, il les assume, et le pronom je, d'élément d'un paradigme, est transmué en une désignation unique et produit, chaque fois, une personne nouvelle. [...] cette expérience n'est pas décrite, elle est là, inhérente à la forme qui la transmet, constituant la personne dans le discours et par conséquent toute personne dès qu'elle parle. [...] La langue pourvoit les parlants d'un même système de références personnelles que chacun s'approprie par l'acte de langage [...]. (Benveniste $1974: 67-68$ )

De même, la confusion est entretenue par des définitions grammaticales qui usent à plusieurs reprises, voire de manière tautologique, de l'emploi du terme « personne »:

\footnotetext{
${ }^{11}$ Selon Benveniste 1966: 265, la $3^{\mathrm{e}}$ personne «n'existe et ne se caractérise que par opposition à la personne $j e$ du locuteur qui, l'énonçant, la situe comme «non-personne ». C'est là son statut. La forme $i l . .$. tire sa valeur de ce qu'elle fait nécessairement partie d'un discours énoncé par « je ». »

${ }^{12}$ Voir la note 9 précédente pour la citation de Benveniste 1966: 263.

${ }^{13}$ Voir Culioli 1999a au sujet de la typographie de Benveniste, et aussi Lyons 1984: 137.
} 
les personnes grammaticales sont «des personnes qui..., à qui..., de qui... $»^{14}$. On se rappelle que le linguiste Marc Wilmet a mis en garde le lecteur de sa grammaire contre la confusion des acceptions du terme «personne». Or, une telle formulation est ambivalente dans la mesure où «la personne qui..., à qui..., de qui...» se comprend dans ce cas comme «être sexué », plutôt que comme «entité grammaticale ayant un référant animé ou inanimé » et se doit d'être clarifiée ${ }^{15}$. Benveniste a recours à de telles définitions $(1966: 252)$ mais il est aussi soucieux d'expliciter les termes de «cette double instance conjuguée : instance de je comme référent, et instance de discours contenant $j e$, comme référé » pour ce qui concerne la $1^{\text {re }}$ personne : «la définition peut alors être précisée ainsi : je est «l'individu qui énonce la présente instance de discours contenant l'instance linguistique je ».» (1966: 252 ; voir aussi 1974 : 82). En l'état, il semble délicat, voire impossible, de conserver le terme «personne » en grammaire sans référer automatiquement aux personnes humaines in praesentia.

La $3^{\mathrm{e}}$ personne, au-delà de l'opposition sémantique animé/inanimé, permet de référer à une entité autre que celle qui parle et à qui l'on parle, parce qu'elle désigne exclusivement ce dont il est parlé. C'est en cela qu'il s'agit d'une entité discursive absente de la scène verbale, ce qui implique qu'elle ne saurait se confondre avec l'un des protagonistes de l'échange. C'est cette altérité, propre au champ situé en dehors de l'espace de l'interlocution, que Benveniste a souhaité mettre au jour en recourant à l'étiquette de non-personne. ${ }^{16}$ Jacquesson soutient, quant à lui, la thèse de Benveniste à l'encontre de «l'abus de langage » qui consiste à parler de la $3^{\mathrm{e}}$ personne et souligne l'intérêt du choix du mot «masque avec son porte-voix » comme terme technique pour désigner les « personnes vraies » :

Dans beaucoup de cas, pourtant, le « sujet de la conversation » n'est pas une personne, puisque aussi bien on peut parler d'une fortune qui s'enfuit ou d'une maladie qui gagne, plutôt que de la fille du barbon local. Cette $3^{\mathrm{e}}$ personne peut être une personne humaine, comme elle peut être la réparation de l'escalier ou la mort. C'est donc par un abus de langage que nous parlons de $« 3^{\mathrm{e}}$ personne ». Les deux seules qui sont à coup sûr de vraies personnes sont celle qui parle et celle qui écoute avant de répondre à son tour. [...]

Si l'on peut s'étonner que les grammairiens anciens (et presque tous les autres jusqu'à nos jours en Europe) aient considéré sans trop d'hésitation que le sujet de la conversation était quelqu'un, une "personne », ou aient fermé les yeux sur l'évident abus de cette fiction, on ne peut en revanche qu'admirer la pertinence du mot «masque » pour désigner l'impermanence $\mathrm{du} \ll \mathrm{je} »$ et $\mathrm{du}$ « tu », puisque dès que «tu » vas répondre, c'est toi qui dira $\ll$ je ». Cela ne montre-t-il pas que le chassé-croisé des fonctions est vraiment un théâtre, et le discours un masque avec son porte-voix, qu'on échange à

\footnotetext{
${ }^{14}$ Voir Arrivé et al. 1986: 495-496 et la position de Wilmet 2003 dans la note 10 précédente.

${ }^{15}$ Voir Wilmet 2003: 293 dans la note 17 suivante.

16 «Il faut garder à l'esprit que la « $3^{\mathrm{e}}$ personne » est la forme du paradigme verbal (ou pronominal) qui ne renvoie pas à une personne, parce qu'elle se réfère à un objet placé hors de l'allocution » Benveniste 1966 : 265. C'est la position adoptée par Maingueneau 1990: 5-7; Maingueneau 1999: 21-28 et aussi par Riegel et al. 2009: 363-36. Hagège 1982: 96 estime que « divers faits confirment cette thèse » mais fait néanmoins remarquer «que "personne" est un terme ambigu » car «il existe des emplois de "tu" ou "il" valant pour "je" (ex. russe parlé) et, dans certaines langues (Hagège 1982: 144 s.), des "ill" de politesse valant "tu". En appelant donc "non-personne" la 3 , on semble suggérer tout ensemble, à tort, que son référent est absent et qu'il n'est pas une personne » Hagège $1982: 97$.
} 
chaque fois pour prendre aussitôt l'autre masque, celui qui a l'air étonné ou inquiet, ou momentanément mué. [...] Et cette comédie des échanges, où l'un prend la pose et l'autre la parole, avant d'inverser les costumes et les rôles, et de recommencer - est si proche du théâtre qu'on ne peut qu'applaudir cette idée de m'appeler une personne, et tu en es une autre. [...]

Notre $3^{\mathrm{e}}$ personne n'est pas une « personne » pour les grammairiens arabes et judéo-arabes, et l'on ne peut qu'approuver, après Benveniste, ce point de vue plus exact. On ne peut que s'étonner de la puissance de nos usages à nous, qui contre tant d'évidence ont maintenu notre préjugé. (Jacquesson 2008 : 23-24; c'est moi qui souligne)

Le «masque avec son porte-voix» du latin-étrusque est donc intrinsèquement lié au sens grec de «vis-à-vis » qui inscrit les «personnes » $\left(1^{\text {re }}\right.$ et $\left.2^{\mathrm{e}}\right)$ dans le cadre de l'interlocution.

De son côté, Maillard (1994: 56-57) a proposé de remplacer le terme de «personne » par celui de «Locution», afin « de nettoyer la catégorie en question de ses «adhérences » idéologiques et de ses nombreux malentendus entraînés par l'opposition benvenistienne «personne »/《non-personne »». Il s'agit alors de parler de l'opposition entre l' « élocutif/allocutif » d'une part et le « délocutif » de l'autre, ce qui revient à opposer le locutoire au non-locutoire ${ }^{17}$. Or, les métatermes «locution » et «allocution», eux-mêmes employés par Benveniste (1966:252-253), ne sont pas encore parvenus à supplanter dans toutes les grammaires ceux de «personne » et de «personnel ».

Quelle que soit la terminologie adoptée, voire revendiquée, la corrélation de personnalité de Benveniste semble incontestable, et a permis la refonte de la classe des pronoms dits «personnels » en deux sous-groupes : les «personnes (vraies)» ou «locutives » sont en fait des non-pronoms, plus couramment appelés «embrayeurs » (ou, rappelons-le, «shifters » en anglais, depuis Jespersen et Jakobson) relevant alors de la catégorie des déictiques (Benveniste 1966 : 252-254), à côté des «pronoms (vrais)» (Benveniste 1966: 255-256) ou «délocutifs », et donc improprement appelés «personnels ${ }^{18}$. Il reste que la terminologie traditionnelle perdure et que pour des facilités d'ordre pédagogique, les grammairiens sont nombreux à continuer de parler de «pronoms personnels » uniformément, contribuant à entretenir les confusions, voire les fusions, entre le grammatical et l'humain, le déictique et l'anaphorique, le locutoire et le non-locutoire. Il s'agit donc d'une posture

\footnotetext{
${ }^{17}$ Maillard n'est pas le seul à se référer au métaterme « locution » et à ses dérivés ; voir Damourette et Pichon cités par Dubois et al. 1994: 357 et Creissels 1995: 122. Wilmet 2003: 293, § 346 recourt à la même terminologie en apportant les précisions suivantes : «L'inventaire selon les personnes donne le résultat suivant.

(1) Première personne ou personne présente locutive (ou élocutive : Creissels 1995) : je, me, moi, nous.

(2) Deuxième personne ou personne présente allocutive (présupposant la première) : tu, te, toi, vous. [...]

(3) Troisième personne ou personne absente délocutive : il(s), elle(s), lui, eux, se, soi, et les pronoms accidentels le, la, les (Cf. §§ 304-305), leur (Cf. § 317). »

18 «La définition ordinaire des pronoms personnels comme contenant les trois termes $j e, t u, i l$, y abolit justement la notion de "personne". Celle-ci est propre seulement à je/tu, et fait défaut dans $i l$. Cette différence foncière ressortira de l'analyse de je. » Benveniste 1966: 251.

On relèvera, ici encore, la présence de guillemets encadrant le terme «personne » indiquant par là qu'il nécessite d'être spécifié linguistiquement parlant : «A quoi donc je se référe-t-il ? A quelque chose de très singulier, qui est exclusivement linguistique : je réfère à l'acte de discours individuel où il est prononcé, et il en désigne le locuteur. » Benveniste 1966: 261.
} 
épistémologique lourde de conséquences, qui privilégie une classification héritée sans l'interroger dans ses fondements, tombés dans l'oubli. En grammaire anglaise, on rencontre le métaterme de «pronoms de discours » ${ }^{19}$ ( $I$ et you) qui présente l'avantage d'être à la croisée des terminologies traditionnelle et linguistique, en ce qu'il désigne d'une part l'appartenance des $1^{\text {re }}$ et $2^{\mathrm{e}}$ personnes à une classe de mots connues sous l'étiquette de "pronoms » et d'autre part le contexte d'emploi dans lequel on les rencontre exclusivement, à savoir le discours, ou encore l'espace de locution/interlocution. On représente ainsi d'une part les partenaires de l'acte énonciatif (les «personnes » < «masques de théâtre » et donc les acteurs présents sur scène uniquement) opposés d'autre part au sujet de la conversation, que ce dernier désigne une entité animée ou inanimée - la fameuse "non-personne ». Il s'agit de la « corrélation de subjectivité », c'est-à-dire de l'autre micro-système du système de la personne chez Benveniste (1966 : 230), qui expose les spécificités « du «je-tu» qui seuls indiquent des personnes $\gg$ :

En effet une caractéristique des personnes «je » et «tu » est leur unicité spécifique : le «je » qui énonce, le «tu » auquel «je »s'adresse sont chaque fois uniques. Mais «il » peut être une infinité de sujets - ou aucun. [...]

Une seconde caractéristique est que «je» et «tu»sont inversibles : celui que «je » définis par «tu » se pense et peut s'inverser en «je », et «je » (moi) devient un «tu ». Aucune relation pareille n'est possible entre l'une de ces deux personnes et «il », puisque «il » en soi ne désigne spécifiquement ni rien ni personne.

Enfin on doit prendre pleinement de cette particularité que la «troisième personne » est la seule par laquelle une chose est prédiquée verbalement. (Benveniste $1966: 230$ )

Chez Benveniste ${ }^{20}$, les métatermes «locution» et «allocution»se rencontrent à côté de celui d' " énonciation », mais c'est ce dernier qui a été retenu pour référer aux courants linguistiques relevant de la théorie de l'énonciation. L'opposition « locutoire / non-locutoire » se comprend-elle dans ce cas comme étant celle de l'énonciatif par rapport au non-énonciatif ? Les termes «énonciateur » et «co-énonciateur » viennentils supplanter ceux de «locuteur» et d' «interlocuteur/allocutaire/co-locuteur », qui sont employés couramment en linguistique, y compris en linguistique énonciative ${ }^{21}$ ? Il ne suffit pas de remplacer un métaterme par un autre, encore faut-il justifier son apport théorique et conceptuel.

\subsection{Ambiguïté des termes « énonciation » et « énonciateur »}

Je souhaiterais terminer ce tour d'horizon critique ${ }^{22}$ de la réception des thèses de Benveniste par la position du linguiste Antoine Culioli, héritier du courant de la linguistique énonciative et fondateur de la Théorie des opérations énonciatives $(\mathrm{TOE})^{23}$. D'après Culioli (1984 : 82-84 [1999a : 121-123]) et Kawaguchi (1984), si

\footnotetext{
${ }^{19}$ Voir Dufaye, Khalifa 2006: 146.

${ }^{20}$ Voir notamment Benveniste 1974: $68 ; 82$.

${ }^{21}$ Voir Ducrot 1982: 74-76.

${ }^{22}$ Nécessairement bref et incomplet. Voir également les critiques de Kerbrat-Orecchioni 2009: 48-50.

${ }^{23}$ Certains auteurs culioliens préfèrent parler de «théorie des opérations prédicatives et énonciatives » (TOPE).
} 
Benveniste a bien mis en évidence «la subjectivité dans le langage » (Benveniste 1966 : 258-266), en particulier à travers l'étude des «personnes (vraies)», ses emplois du terme «énonciation » entretiennent la confusion entre deux niveaux d'analyse distincts : le plan de la «mécanique interlocutoire »(Culioli 1990 : 130) et le plan du métalinguistique qui seul relève de l'énonciatif. Comme Ducrot, mais avec des définitions différentes dans le cadre de l'emploi d'une terminologie en apparence identique («locuteur»; «énonciateur»), Culioli remet en cause «l'unicité du sujet parlant» (Ducrot 1982: 65), appelé indifféremment locuteur ou énonciateur par Benveniste $^{24}$ et à sa suite par les linguistes énonciativistes. L'énonciation ne saurait se confondre avec la locution, à savoir le cadre de l'échange verbal réduit exclusivement au dialogue, dans lequel les «partenaires » (Benveniste $1966: 263 ; 1974: 77 ; 85$ ) de l'interlocution, je/tu, dans la «situation d' «allocution»»(Benveniste $1966: 253$ ), sont nécessairement présents physiquement tandis que $i l$ en est absent, et ainsi mis hors locution. Ainsi, il importe, selon Culioli, de bien les distinguer et de leur donner un statut défini dans la métalangue: le sujet parlant désigne le locuteur, à savoir l'individu qui articule matériellement les sons de la chaîne parlée, et qui s'adresse à l'interlocuteur en tant que récepteur du message présent face au locuteur, tandis que l'énonciateur se distingue du locuteur en renvoyant à une instance abstraite d'ordre symbolique qui est le repère de la prise en charge de l'énoncé, à savoir l'entité métalinguistique ${ }^{25}$ qui s'en porte garante dans une relation d'altérité par rapport au co-énonciateur. L'énonciateur est le support des modalités ${ }^{26}$ et des affects dans l'énoncé, et donc l'origine ou la source énonciative. Le co-énonciateur, quant à lui, est la représentation que l'énonciateur se fait de soi-même ou d'autrui en tant cible énonciative de l'énoncé. Quant au support de la prédication, dénommé sujet syntaxique traditionnellement, Culioli accepte de le désigner à la rigueur comme sujet de l'énoncé, mais réserve le terme de sujet à celui d'énonciateur, distinct du locuteur. Il pointe alors vers «un concept manquant» chez Benveniste et précise que «le concept qui manque est le concept d'énonciateur, tel que je l'entends » (Culioli 1984 : 83 [1999a : 121]). Selon lui, Benveniste «privilégie le locuteur et la production » (Culioli 1984 : 84 [1999a: 122]). Afin de pallier ce manque, Culioli distingue (au moins) trois instances de sujet ${ }^{27}$, qu'il note respectivement $\mathrm{S}_{0}, \mathrm{~S}_{1}, \mathrm{~S}_{2}$ dans le cadre de son système métalinguistique. Dans l'article précédemment cité de 1984, il est question de $S_{1}$ et $S_{2}$ : «Si nous notons $S_{2}$ le terme je et $S_{1}$ le locuteur, nous nous apercevons que, derrière les instances discrètes de locution, individuelles, et qui n'ont

\footnotetext{
${ }^{24}$ «L'acte individuel par lequel on utilise la langue introduit d'abord le locuteur comme paramètre dans les conditions nécessaires à l'énonciation » Benveniste 1974: 81. On note là l'emploi de « locuteur » à côté de deux emplois de «énonciateur» dans la suite de l'article sans changement de sens : «Outre les formes qu'elle commande, l'énonciation donne les conditions nécessaires aux grandes fonctions syntaxiques. Dès lors que l'énonciateur se sert de la langue pour influencer en quelque manière le comportement de l'allocutaire, il dispose à cette fin d'un appareil de fonctions ", à savoir l'« interrogation », et aussi l'« intimation »: «ordres, appels conçus dans des catégories comme l'impératif, le vocatif, impliquant un rapport vivant et immédiat de l'énonciateur à un autre dans une référence nécessaire au temps de l'énonciation » Benveniste 1974: 84.

${ }^{25}$ Kawagushi 1984: 122 critique la position de Benveniste (ci-après noté B.) en ces termes : «La tentative benvenistienne de fonder la catégorie de la personne à partir du sujet locuteur en s'appuyant sur des morphèmes ne peut se justifier que si ces morphèmes sont rapportés à un paramètre métalinguistique. On est donc fondé à dire que les termes $j e$ et $t u$ sont en fait, contrairement à ce qu'en dit B., des emplois métalinguistiques notant des sujets énonciateur et co-énonciateur. »

${ }^{26}$ Voir Filippi-Deswelle 2008 (à paraître) et 2012a, pour une présentation détaillée de la position de Culioli sur le concept de « sujet énonciateur».

${ }^{27}$ Voir Culioli 1990: 152-155 au sujet de l'analyse de bien et des associations/dissociations entre énonciateur, locuteur et sujet d'énoncé.
} 
de référence qu'actuelle, il doit exister un centre, un repère origine qui assure la stabilité du système de repérage » (Culioli 1984 : 83 [1999a : 121]), ainsi défini :

ce système se caractérise par des localisations en cascade : une relation prédicative (elle-même issue d'un ensemble de relations de repérage) est localisée par rapport à un index d'événement, qui tire sa valeur de son repérage par rapport au moment de locution, lui-même repéré par rapport à un repère-origine (repère absolu). A partir du repère absolu, on pourra construire un repère translaté et un repère décroché (repère fictif). [...] Si l'on se tourne maintenant vers le concept d'énonciateur, on notera que l'énonciateur est dans une relation d'altérité au co-énonciateur telle que l'on puisse avoir coalescence ou séparation. Au contraire, le locuteur et l'interlocuteur sont toujours séparés et l'on ne doit pas confondre le champ intersujets (où sujet renvoie à énonciateur) avec la mécanique interlocutoire. (Culioli 1990 : 130)

Il s'opère ainsi un «calcul sur le dispositif paramétré de repères situationnels » : « $\mathrm{Sit}_{0}$ est le repère-origine, $\mathrm{Sit}_{1}$ le repère de locution, etc. ; les paramètres concernent la catégorie $\mathrm{S} \Leftrightarrow \mathrm{S}$ et la catégorie $\mathrm{T} \Leftrightarrow \mathrm{T} »$ (Culioli 1990, note 4, p. 137). La situation d'énonciation peut ou non coïncider avec la situation de locution (1990: 148-149), $\mathrm{Sit}_{1}$, ou la situation de l'événement (énoncé), $\mathrm{Sit}_{2}$. « $\mathrm{S}_{2}$ note le sujet de l'énoncé » (1990 : 151). La construction d'un « repère fictif » permet de « dissocier l'énonciateur du locuteur (ou scripteur)» (1990: 150): on parle alors de «l'énonciateur fictif, $\mathrm{S}_{0}{ }^{1}$ » $(1990: 151)$. Le tout peut s'écrire selon la formule synthétique suivante : $\lambda \underline{\varepsilon}<\operatorname{Sit}_{2}\left(\mathrm{~S}_{2}, \mathrm{~T}_{2}\right) \underline{\varepsilon} \operatorname{Sit}_{1}\left(\mathrm{~S}_{1}, \mathrm{~T}_{1}\right) \underline{\varepsilon} \operatorname{Sit}_{0}\left(\mathrm{~S}_{0}, \mathrm{~T}_{0}\right)>($ Culioli 1999a [1982] : 105) ${ }^{28}$. Une telle approche permet notamment de traiter des problèmes de discours indirect ${ }^{29}$. Maillard (1994: 65) adresse d'ailleurs une critique du même type à Benveniste et à Joly en invitant ce dernier à «un abandon de l'analyse benvenistienne de $j e$, qui confond indûment l'indice formel et son designatum ». Il précise en effet que :

la chose est claire quand Benveniste affirme : «Je est l'individu qui énonce la présente instance de discours » (P.L.G., 1966, p. 252). Non seulement je n'est pas un individu - il doit être distingué de son référent - mais c'est un tort de le confiner dans «la présente instance de discours » comme s'il n'existait pas de discours rapporté : «Paul m'a dit : je vais au marché ». (Maillard $1994: 65$ )

Cette forme de confusion, d'ambiguïté, associée aux emplois du terme d' «énonciation» me font préférer la dénomination de "Théorie des opérations (prédicatives et) énonciatives » pour caractériser les travaux de Culioli à celle retenue par l'éditeur de ses articles dans les trois volumes intitulés Pour une linguistique de l'énonciation parus chez Ophrys (1990, 1999a et 1999b). En effet, il s'agit d'une conception dynamique du langage où tout élément est construit et non donné, et rapporté à une instance subjective organisatrice et responsable de ses énoncés. La disjonction entre les fonctions de locuteur et d'énonciateur sera manifestée par la présence de marqueurs venant moduler l'assertion, comme l'inversion interrogative qui suspend la prise en charge énonciative et recourt au co-énonciateur pour trancher,

\footnotetext{
${ }^{28}$ A compléter par Culioli 1999a : $130 ; 167$.

${ }^{29}$ Voir Kawagushi 1984: 120.
} 
ou un modal qui nuance les degrés de certitude liés à la réalisation de la prédication. Le concept d'énonciateur est donc indissociable d'une théorie de l'assertion d'une part, et du champ de forces inter-sujets dont le co-énonciateur est partie prenante, même en cas d'absence d'interlocuteur réel dans la situation d'énonciation. Comme on le voit, dans une telle conception métalinguistique de la personne ou du sujet, on se tient éloigné de toute confusion avec le référent de je ou $t u$.

Culioli (1984 : 83 [1999a : 121]) fait remarquer que Benveniste avait pressenti ces développements («le problème est perçu ») sans pour autant les avoir explicités dans ses travaux. Chez Benveniste, il est indiqué que :

on peut utiliser la $2^{\mathrm{e}}$ personne en dehors de l'allocution [...] Il faut et il suffit qu'on se représente une personne autre que «je » pour qu'on lui affecte l'indice «tu ». Ainsi toute personne qu'on se représente est de la forme «tu », tout particulièrement - mais non nécessairement - la personne interpellée. Le «tu » (« vous ») peut donc se définir : «la personne non-je». (Benveniste $1966: 232$ )

La conception du «co-énonciateur» chez Culioli permet de théoriser cette dissociation entre $t u$ et "l'allocutaire $»^{30}$, et d'en proposer un traitement sur le plan imaginaire, comme l'indique Kawagushi (1984: 125). Ce dernier adresse par ailleurs une autre critique à l'approche benvenistienne de l'interlocuteur, désigné comme «personne non-subjective » par Benveniste (1966: 232) et dont le « rôle se limite à une simple présence passive» (Kawagushi 1984: 123). Selon cet auteur, «c'est toujours le locuteur qui à un moment donné prend en charge son énoncé. Mais dans l'énonciation l'interlocuteur est aussi présent. De diverses façons celui-ci participe à la construction de l'énoncé » (Kawagushi 1984 : 124). J'ajoute, dans une perspective culiolienne, qu'il ne s'agit pas ici uniquement de «présence» physique de l'interlocuteur mais surtout de la prise en compte métalinguistique du co-énonciateur dans le calcul des repérages, ce qui nécessite un ajustement de sujet à sujet ${ }^{31}$ :

Le système de référence n'est pas fourni tout constitué, mais est construit par un sujet qui est partie intégrante du système. Ainsi, on n'a pas affaire à un observateur extérieur, muni d'un référentiel objectivable, mais tout se passe

\footnotetext{
${ }^{30}$ Dans les termes de Benveniste 1974: 82, la dissociation entre tu et l' «allocutaire » s'appréhende ainsi : "Mais immédiatement, dès qu'il se déclare locuteur et assume la langue, il implante l'autre en face de lui, quel que soit le degré de présence qu'il attribue à cet autre. Toute énonciation est, explicite ou implicite, une allocution, elle postule un allocutaire. » On aura noter, une fois de plus, l'emploi des italiques, auxquels il convient, selon Culioli, de donner un statut métalinguistique.

${ }^{31}$ Culioli précise qu'il convient de prendre en compte «un, le concept de régulation; deux, l'intersubjectivité mais avec la construction du co-énonciateur, qui est le miroir de l'énonciateur (pas du co-énonciateur extérieur qu'est l'interlocuteur) et, en même temps - je pense que c'est lié au second point d'ailleurs - une conception qui m'a fait sortir du "discours intérieur", qui est au fond un soliloque ; c'est-à-dire que, si on a un énonciateur / co-énonciateur, toute cette activité n'est jamais une activité solitaire »Culioli, Normand 2005: 155-156. On assiste ici à la théorisation par Culioli des propos de Benveniste 1974: 85-86 sur le «monologue », en tant que « dialogue intériorisé, formulé en "langage intérieur", entre un moi locuteur et un moi écouteur. Parfois le moi locuteur est seul à parler ; le moi écouteur reste néanmoins présent; sa présence est nécessaire et suffisante pour rendre signifiante l'énonciation du moi locuteur. »

L'activité de langage, quant à elle, est liée à ce que Culioli appelle l'activité épilinguistique du sujet qui s'entend parler et peut insister sur le bien-fondé de ses formulations, et même les rectifier si nécessaire, à côté de l'activité métalinguistique consciente du linguiste. Voir aussi Filippi-Deswelle 2012b au sujet de la notion d'ajustement dans la TOE d'Antoine Culioli, en particulier « Pour (ne pas) conclure. Pour une linguistique des ajustements énonciatifs ».
} 
comme si le sujet se constituait comme origine du système de référence. Or, ce sujet doit construire le système par rapport à un autre sujet à qui il veut faire partager sa représentation; situation complexe, car, en dehors du pointage (lorsqu'on a affaire à du visible et que l'interlocuteur est présent), nous ne possédons pas de référentiel externe et pré-ajusté qui fonctionnerait de sujet à sujet.

Il faut donc construire un système de référence stable et ajustable, qui permette à autrui de reconstruire, à partir d'énoncés, et le système de référence et l'opération complexe de référenciation, grâce à quoi le texte sera interprété et muni de valeurs référentielles. (Culioli 1999b : 167)

Ainsi, d'après Culioli (1984 [1999a]), de Vogüé (1992) et Culioli et Normand (2005 : 157 ; 162-164), il n’y a pas de théorie du sujet énonciateur chez Benveniste, dans la mesure où ce dernier "ramène l'activité de représentation à un acte de locution » (Culioli (1984:84 [1999a : 122]).

Le concept de «personne » doit donc être dissocié de celui de «sujet » entendu comme énonciateur/co-énonciateur. En effet, la personne relève d'une part du sujet anthropologique doué de conscience, de pensée, de sentiment, et de langage, et aussi doté d'un corps, pris dans une culture et une langue données. Dans ce cas, le sujet est à la croisée de ce que Culioli (Culioli et Normand 2005 : 164-166) appelle le «transindividuel » (héritage des pratiques socio-culturelles et linguistiques des sujets) et l' « interpersonnel » (la possibilité de se mettre à la place d'une autre personne). D'autre part, la personne s'entend comme position grammaticale (par rapport à d'autres) dans le cadre de la prise en parole, en tant que sujet de l'énoncé $\left(\mathrm{S}_{2}\right)$. Le sujet humain est selon les cas locuteur $(j e)$, interlocuteur $(t u)$ ou situé en dehors de l'allocution $(i l)$. Mais le sujet énonciateur $\left(\mathrm{S}_{0}\right)$ est construit à partir du locuteur $\left(\mathrm{S}_{1}\right)$ comme centre organisateur de l'énonciation, en tant qu'origine énonciative d'ordre métalinguistique, dans le champ des relations intersubjectives « de soi à soi » ou « de soi à un autre que soi » qui, selon Culioli, «peut renvoyer à soi et qui peut renvoyer à l'autre » (Culioli et Normand $2005:$ 165). En résumé,

Ce qui existe dans $j e, t u, i l$, c'est la forme qui fait qu'il y a une origine : il y a une branche qui va d'un côté aller à autre que l'origine, et en même temps, il $\mathrm{y}$ a toujours une autre branche qui peut se ramener à une boucle; et puis après vous avez ce qui n'est ni le premier, ni le second, mais qu'entre l'origine et la non-origine vous avez une zone frontière où se situe le coénonciateur qui va pouvoir être ramené à l'origine ou à une sorte d'intentio, de représentation d'autrui ; ça, on l'a de toute façon. [...] Et les positions dont je parlais sont des repères, des repères par rapport à. (Culioli et Normand 2005 : 167-168)

Danon-Boileau (2007) fait la synthèse de la théorie de Culioli sur la personne qui réélabore les corrélations de personnalité et de subjectivité en termes d'instances formelles en relations de repérage, où l'opérateur de repérage prend quatre valeurs possibles (égal, différent, indéfini ${ }^{32}$, en rupture) :

\footnotetext{
${ }^{32}$ Danon-Boileau 2007: 26 ajoute que «si la valeur de l'opérateur de repérage est « indéfini », autrement dit si la référence du sujet dans l'énoncé peut occuper, par rapport à celle de l'énonciateur, n'importe laquelle des trois positions précédentes, le pronom qui résulte est « on» (qui défait l'opposition je/tu sans la confondre en une «première personne » comme fait « nous »). » Voir aussi
} 
Culioli a souvent insisté sur le fait que les pronoms je, tu, il, ne renvoient pas à des entités en soi, mais sont des valeurs qui indiquent la situation d'entités par rapport à l'énonciateur. Ils sont les images de l'état de la relation entre l'énonciateur et «autre chose» (la référence associée au sujet dans l'énoncé). Cela revient à dire que le système ne définit pas les pronoms comme des billes de plus en plus éloignées d'un centre constitué par l'énonciateur mais comme des valeurs du rapport énonciateur - «autre chose », ce qui est une banalité que l'on a tendance à oublier mais que la différence entre «tu» et «il » vient opportunément rappeler. [...] dans l'application de l'opérateur de repérage au système des pronoms, il y a l'énonciateur face à un sujet qui ne commute pas avec lui (le «il», hors système), et l'énonciateur face à un autre sujet ; cette seconde position, la position «en système » se subdivise encore en un «égal» (je) et un « différent » (tu). (Danon-Boileau 2007 : 30-32)

\subsection{Métalangue et données empiriques : quel ajustement ?}

En conclusion de cette deuxième partie, je souhaiterais attirer l'attention sur le risque inhérent à toute métalangue d'engendrer des confusions, et en particulier sur le risque qui consisterait à confondre énonciateur et locuteur en toutes circonstances dans le cadre de l'utilisation du métaterme d'énonciateur dans la TOE. Dans les lignes précédemment citées, cette distinction est neutralisée car elle renvoie à une relation de repérage implicite entre les trois instances qui sont en effet le plus souvent confondues dans une relation d'identification dans les assertions : dans l'exemple (1) déjà analysé, je est l'image du locuteur $\left(\mathrm{S}_{1}, 1\right.$ 'animé qui dit le contenu propositionnel, mis en relation avec le référent extralinguistique, François ou Catherine) et de l'énonciateur ( $\mathrm{S}_{0}$, qui prend en charge le jugement émis sur Jules), en plus d'être le sujet de l'énoncé $\left(\mathrm{S}_{2}\right.$, à propos duquel il est dit quelque chose, à savoir < moi - ne pas

Groussier, Rivière 1996: 146 qui, comme Maingueneau 1990 ; 1999 et Jacquesson 2008, revisitent la prétendue uniformité des «pronoms personnels »: «La catégorie verbale de la personne regroupe des marqueurs de détermination d'un prédicat par repérage par rapport soit à l'énonciateur $\left(\mathrm{S}_{0}\right)$ les $1^{\mathrm{re}}$ et $2^{\mathrm{e}}$ personnes, soit au couple énonciatif entier $\left(S_{0}\right.$ et $\left.S_{1}\right)$ pour la $3^{\mathrm{e}}$ personne. Lorsqu'il y a accord selon la personne entre la forme verbale et le $1^{\text {re }}$ argument, les marques de personne servent aussi à indiquer partiellement l'opération de prédication.

1) Les pronoms personnels français et anglais renvoyant à des fonctions énonciatives sont : a) le pronom de $1^{\text {re }}$ personne du singulier je/moi/me et $I / m e / m y$ qui renvoient à l'énonciateur. Au pluriel, le pronom de $1^{\text {re }}$ personne nous ou welus/our renvoie à un groupe incluant l'énonciateur et éventuellement le coénonciateur («nous inclusif»). b) le pronom de $2^{\mathrm{e}}$ personne you/your renvoie à un coénonciateur soit unique soit multiple en anglais, tu/toi/te renvoyant à un coénonciateur unique et familier et vous à un coénonciateur unique et non-familier ou multiple en français.

2) Les pronoms anaphoriques de $3^{\mathrm{e}}$ personne renvoient à tout ce qui est extérieur au couple énonciatif. Ainsi, le système des pronoms personnels manifeste trois des valeurs de la relation de repérage : identification à $S_{0}($ valeur $=)$ pour la $1^{\text {re }}$ personne, différence par rapport à $S_{0}($ valeur $\neq)$ pour la $2^{\mathrm{e}}$ personne, non-repérage par rapport à la Situation d'énonciation et à l'ensemble du couple énonciatif (valeur $\omega$ ) pour la $3^{\mathrm{e}}$ personne. C'est pourquoi la valeur référentielle des pronoms de $3^{\mathrm{e}}$ personne est établie par anaphore c'est-à-dire tributaire d'une première mention de l'entité désignée dans un énoncé précédent ou antérieurement dans le même énoncé. C'est parce qu'ils sont ainsi définis et non directement par rapport à la Situation d'énonciation que les pronoms de $3^{\mathrm{e}}$ personne ont, en français comme en anglais, des formes différentes selon le genre, du moins au singulier : il/le/lui et he/him/his pour le masculin, elle/la/lui et she/her pour le féminin, it/its pour le neutre en anglais. Le pluriel anglais they vaut pour les trois personnes alors que le français a ils/les/eux au masculin et elles/les au féminin. » 
sentir Jules>). Mais il faut la garder à l'esprit afin de ne pas la dévoyer, ni passer à côté de son caractère opératoire dans d'autres contextes syntactico-énonciatifs, notamment lors de l'emploi de plusieurs occurrences de je au sein d'un même énoncé, comme en (7) :

(7) Et quand je dis qu'il s'est fait battre à plate couture, je veux vraiment dire qu'il s'est fait battre à plate couture, plate couture de chez « plate couture », et je n'emploie pas ce mot dans n'importe quel sens... (Culioli 2002 : 28)

Le premier je, sujet de l'énoncé $\left(\mathrm{S}_{2}\right)$ associé au prédicat dire, est la trace du locuteur $\left(\mathrm{S}_{1}\right)$, le deuxième je associé au prédicat complexe vouloir dire est celle de l'énonciateur-asserteur ${ }^{33}$ ( $\mathrm{S}_{0}$, qui prend en charge), et la troisième occurrence associée au prédicat employer un mot indique encore la subjectivité consciente et assumée de l'énonciateur $\mathrm{S}_{0}$, responsable de ses choix énonciatifs. Il s'agit d'une réflexion d'ordre métalinguistique où je n'est pas tant le sujet grammatical du prédicat (ce qu'il est aussi nécessairement sur le plan syntaxique) que le représentant de l'instance subjective origine, organisatrice et constructrice de l'énoncé analysant son travail énonciatif. On retrouve là les étapes typiques de l'opération d'assertion selon Culioli, « je tiens à dire que je pense que $\langle\mathrm{S}$ - P $>$ est le cas » (Culioli $1990: 131-132$ ) - mais ici appliquées de manière réfléchie à la pratique énonciative de l'énonciateur détaillées comme suit sur le plan métalinguistique, alors qu'elles sont le plus souvent implicites sur le plan épilinguistique ${ }^{34}$ :

Cette dernière <l'assertion il a accepté> comporte (1) un engagement, une prise en charge (je tiens à, je veux), (2) une matérialisation (dire, écrire, bref faire exister de façon perceptible), (3) une instance de représentation (selon le cas : je pense, je crois, je sais), (4) une représentation, c'est-à-dire une occurrence notionnelle que l'énonciateur situe par rapport à un espace de référence («qu'il a accepté est le cas »). En résumé, on obtient je tiens à dire que je pense (etc) que <p> est le cas. (Culioli 1999b : 96)

L'assertion est produite en vue d'être interprétée, selon ce que Culioli appelle « la double contradiction fondamentale de l'énonciation, entendue au sens de production et reconnaissance interprétative des textes $»$ :

Tout énoncé suppose un acte dissymétrique d'énonciation, production et reconnaissance interprétative. Ramener l'énonciation à la seule production et l'énonciateur au locuteur, c'est, en fin de compte, ne pas comprendre que l'énoncé $n$ 'a pas de sens sans une double intention de signification chez les

\footnotetext{
${ }^{33}$ Voir Filippi-Deswelle 2012a concernant l'approfondissement de l'étude du statut de l'asserteur, dont la présente contribution constitue le premier volet.

${ }^{34}$ L'exemple (7), extrait de Culioli 2002: 27-28, est précédé des propos suivants : « Notre activité mentale est une activité qui continue, à mon avis - un avis fondé sur les observations, mais je n'irai bien évidemment pas jusqu'à dire : voilà comment cela fonctionne - de telle manière qu'elle suppose un travail incessant en dehors même de notre position de locuteur; si j'ai introduit le terme d'énonciateur et de co-énonciateur, c'est en partie pour cette raison : c'est parce que nous n'avons pas une activité de langage simplement quand nous parlons avec autrui. » L'énonciateur a donc à voir avec ce qu'A. Culioli appelle l'activité épilinguistique, activité métalinguistique permanente, spontanée et non maîtrisée des sujets (par opposition à l'activité métalinguistique, consciente, produite et maîtrisée par le linguiste, chargée précisément de capter « le travail enfoui de l'activité énonciative », l'activité épilinguistique des sujets parlants). Exemple <correspondant à notre exemple (7)> ». (Michel Viel, auteur de la note 26, p. 28 in Culioli 2002: 27-28.
} 
énonciateurs respectifs. Ces derniers sont à la fois émetteur et récepteur, non point seulement en succession, mais au moment même de l'énonciation. En bref la communication à valeur référentielle strictement externe et explicite n'est qu'un cas limite. (Culioli 1999b:47, note 6 ; cette citation synthétise les critiques de Culioli à l'égard de Benveniste)

A l'encontre d'un schéma symétrique présent chez Benveniste entre le locuteur et l'allocutaire, Culioli insiste sur la dissymétrie au sein du couple énonciateur/coénonciateur. Ceci est particulièrement perceptible dans les cas où le sujet a l'impression de s'entendre parler, et peut ainsi par la suite rectifier ses propos (par exemple à l'aide d'expressions adverbiales comme pour autant ou en tout cas en français et though ou anyway en anglais ${ }^{35}$ ). Dissymétrie et distance réflexive participent ainsi d'une conception de l'activité de langage, à la fois symbolique et signifiante, qui fait la part belle à l'ajustement inter-sujets, de type intra- et/ou intersubjectif. Cet ajustement peut se matérialiser par l'emploi de la $2^{\mathrm{e}}$ personne en regard de celle de la $1^{\text {re }}$ lorsque l'on veut gloser cette activité dialogale interne au sujet ${ }^{36}$ que l'on désigne par exemple en psychologie par le terme de «critique intérieur » où $t u$ est l'image intériorisée de la voix parentale, comme c'est le cas en (8) :

(8) Je $\left(\mathrm{S}_{1}\right.$ identifié à $\left.\mathrm{S}_{0}\right)$ sens que je $\left(\mathrm{S}_{2}\right)$ vais avoir du mal à boucler ma communication dans les temps. Oui, tu (relation de soi à soi entre l'énonciateur $\mathrm{S}_{0}$ et le co-énonciateur $\mathrm{S}_{0}{ }_{0}$ ) gères mal ton temps. Enfin bon / En tout cas, je $\left(\mathrm{S}_{2}\right.$ identifié à $\mathrm{S}_{1}$ identifié à $\left.\mathrm{S}_{0}\right)$ vais faire au mieux dans le temps qu'il me reste.

Les personnes sont donc en relation entre elles ( $1^{\text {re }}$ et $2^{\mathrm{e}}$ pers.), et aussi avec la nonpersonne ( $3^{\mathrm{e}}$ pers.), sans fusion ni confusion, mais dans un rapport constant d'interactions de type inter- ou extra-personnel. Le système de la personne montre que l'altérité est constitutive de l'identité du sujet, et que si opposition il y a, c'est d'une dissociation associative qu'il s'agit : je ne se comprend que par rapport à $t u$ et à il/elle dans un rapport de complémentarité à reconstituer pour chaque situation d'énonciation, intra- ou inter-subjective.

\section{Les personnes au pluriel fusionnent-elles?}

Que les personnes et la non-personne au singulier soient bien délimitées, bien distinctes les unes des autres, semble être inscrit dans la marque de nombre qui leur est associée, ainsi que dans la signification même du nombre singulier qui renvoie à l'unique sur le plan référentiel. Qu'en est-il au pluriel ? L'opération de pluralisation

\footnotetext{
${ }^{35}$ Voir Filippi-Deswelle 2009 ; 2010 ; 2012c.

${ }^{36}$ Voir déjà chez Benveniste les germes d'une telle analyse en termes de «moi locuteur» et «moi écouteur » : «Parfois aussi le moi écouteur intervient par une objection, une question, un doute, une insulte. La forme linguistique que prend cette intervention diffère selon les idiomes, mais c'est toujours une forme "personnelle". Tantôt le moi écouteur se substitue au moi locuteur et s'énonce donc comme "première personne"; ainsi en français où le "monologue" sera coupé de remarques ou d'injonctions telles que : "Non, je suis idiot, j'ai oublié de lui dire que...". Tantôt le moi écouteur interpelle à la “deuxième personne" le moi locuteur : "Non, tu n'aurais pas dû lui dire que...". » Benveniste 1974: 86. Et de nouveau, on aura noté l'emploi benvenistien des guillemets pour «monologue » et «personnelle », signalant un métaterme à définir dans le premier cas, et un métaterme spécifique à la linguistique de Benveniste dans le second, comme référant à la corrélation de personnalité.
} 
rime-t-elle avec addition, voire fusion, d'éléments mis dans le même panier, et par là confondus en une masse homogène, indifférenciée ? Jacquesson (2008:29-30), après bien d'autres, dénonce ici un autre «abus de langage », et même «la dimension truquée du pluriel ».

\subsection{La question du pluriel et sa mise en question}

Il est en effet établi depuis longtemps que la mise au pluriel des première et deuxième personnes du singulier ne signifie pas leur simple multiplication ${ }^{37}$. Selon Jacquesson (2008: 30), «pour «vous » et pour «nous », il ne s'agit pas d'un vrai pluriel mais d'un trait associatif: une association, souvent toute virtuelle, dont le singulier correspondant « toi », « moi », est le pivot. » Le caractère de pronoms vrais, proche des noms, de la $3^{\mathrm{e}}$ personne, explique que le pluriel s'aligne morphologiquement sur celui des formes du pl. des noms, par l'ajout de la désinence $-s$ : il > il-s; elle > elle-s, sans changer de radical. Il n'en va pas de même pour les $1^{\text {re }}$ et $2^{\mathrm{e}}$ personnes, comme le rappelle Jacquesson (2008: 156), contrairement à ce qu'indique la morphologie de nous et de vous à première vue :

Prenons l'exemple du français, où les pronoms «pluriel» nous et vous sont effectivement pourvus d'un $-s$ du pluriel : cela n'empêche pas de voir que les pronoms singuliers correspondants ne sont nullement *nou et *vou, mais je et $t u$. Il faut donc bien admettre que la relation qui existe entre je et nous, et entre $t u$ et vous est d'un tout autre ordre que celle qui existe entre un singulier et un pluriel. Mais il faut bien admettre aussi que la langue ellemême, et depuis très longtemps comme on verra, a sur-marqué cette relation par la marque ordinaire de pluriel. On peut dire aussi que les locuteurs, depuis plusieurs millénaires dans ce cas, ont « réanalysé », en les considérant désormais comme des pluriels, les mots nous et vous, et c'est cette réanalyse qui est patente dans l'emploi du $-s$ de ces deux pronoms. (Jacquesson 2008 : 156)

On notera d'ailleurs que si la marque $-s$ est présente dans les (pronom conjoint en fonction objet direct) et dans eux/elles (pronoms disjoints, avec la variante $-x$ au masc.), elle disparaît dans leur (pronom conjoint en fonction objet indirect). Ici encore, le paradigme formel est loin d'être homogène. Ainsi, la pluralisation se décline sur le mode associatif du groupement, de la «jonction» selon Benveniste, loin de toute fusion ou confusion, au moins aux $1^{\text {re }}$ et $2^{\mathrm{e}}$ personnes :

\footnotetext{
${ }^{37}$ Voir Meillet 1937: 331, cité par Jacquesson 2008: 189, qui signalait déjà en tête de ses remarques sur les pronoms personnels indo-européens, que «d'une part le singulier et de l'autre le pluriel (et le duel) d'une seule et même personne sont notés par des mots distincts : latin ego et $n \bar{o} s, t \bar{u}$ et $u \bar{o} s$. C'est qu'en effet le pluriel a ici un sens spécial : nōs signifie "moi et d'autres", et non plusieurs "moi". » Voir aussi Benveniste 1966: 235 : "D’une manière générale, la personne verbale au pluriel exprime une personne amplifiée et diffuse. Le "nous" annexe au "je" une globalité indistincte d'autres personnes. Dans le passage du "tu" à "vous", qu'il s'agisse du "vous" collectif ou du "vous" de politesse, on reconnaît une généralisation de "tu", soit métaphorique, soit réelle, et par rapport à laquelle, dans des langues de culture surtout occidentales, le "tu" prend souvent valeur d'allocution strictement personnelle, donc familière. Quant à la non-personne ( $3^{\mathrm{e}}$ personne), la pluralisation verbale, quant elle n'est pas le prédicat grammaticalement régulier d'un sujet pluriel, accomplit la même fonction que dans les formes "personnelles": elle exprime la généralité indécise du on (type dicunt, they say). C'est la non-personne même qui, étendue et illimitée par son expression, exprime l'ensemble indéfini des êtres nonpersonnels. Dans le verbe comme dans le pronom personnel, le pluriel est facteur d'illimitation, non de multiplication. »
} 
On sait bien que, dans les pronoms personnels, le passage du singulier au pluriel n'implique pas une simple pluralisation. [...] Dans la grande majorité des langues, le pluriel pronominal ne coïncide pas avec le pluriel nominal, du moins tel qu'on le représente ordinairement. Il est clair en effet que l'unicité et la subjectivité inhérentes à «je» contredisent la possibilité d'une pluralisation. S'il ne peut y avoir plusieurs « je » conçus par le même «je » qui parle, c'est que «nous» est, non pas une multiplication d'objets identiques, mais une jonction entre «je» et le «non-je», quel que soit le contenu de ce «non-je ». Cette jonction forme une totalité nouvelle et d'un type tout particulier, où les composantes ne s'équivalent pas : dans «nous », c'est toujours «je » qui prédomine puisqu'il n'y a de « nous » qu'à partir de «je», et ce «je» s'assujettit l'élément «non-je» de par sa qualité transcendante. La présence du « je » est constitutive du «nous ». (Benveniste $1966: 233)$

Il est également bien connu que nous par exemple peut se comprendre comme étant « inclusif » $\left(1^{\text {ère }}+2^{\text {ème }}\right.$ pers. $)$ ou « exclusif » $\left(1^{\text {ere }}+3^{\text {ème }}\right.$ pers. $)$, désignés comme tels « faute de mieux » par Benveniste :

Plus importante nous parait l'analyse de cette catégorie « inclusif-exclusif » au point de vue des relations de personne [...] Ces deux pluralisations de la $1^{\mathrm{re}} \mathrm{sg}$. servent à conjoindre dans chaque cas les termes opposés des deux corrélations qui ont été dégagées. Le pluriel exclusif ( « moi + eux ») consiste en une jonction des deux formes qui s'opposent comme personnelle et nonpersonnelle en vertu de la «corrélation de personne ». [...] dans «nous» inclusif qui s'oppose à « lui, eux », c'est « toi » qui ressort, tandis que, dans « nous » exclusif qui s'oppose à « toi, vous », c'est « moi » qui est souligné. Les deux corrélations qui organisent le système des personnes au singulier se manifestent ainsi dans la double expression de «nous ». (Benveniste 1966 : 234)

On retrouve ainsi la problématique de la dissociation associative propre au singulier des $1^{\text {re }}$ et $2^{\mathrm{e}}$ personnes et de la non-personne ( $3^{\mathrm{e}}$ pers.). Dans ce cas, la pluralisation s'effectue par un groupement repéré par rapport à je, soit de je avec tu (ou tu et d'autres comme toi), soit de je avec il/elle (ou il/elle et d'autres comme lui/elle), voire de je avec tu (ou tu et d'autres comme toi) et avec il/elle (ou il/elle et d'autres comme lui/elle).

Il existe aussi des emplois où nous n'a pas de référence multiple mais renvoie en fait à l'unique, et donc à je selon un point de vue différent, comme l'indique Benveniste :

Ce «nous» est autre chose qu'une jonction d'éléments définissables; la prédominance de «je»y est très forte, au point que, dans certaines conditions, ce pluriel peut tenir lieu de singulier. La raison en est que «nous » n'est pas un « je » quantifié ou multiplié, c'est un «je»dilaté audelà de la personne stricte, à la fois accru et de contours vagues. (Benveniste 1966 : 234-235)

Je m'interrogerai, pour finir, sur le pluriel de modestie.

\subsection{Le « nous » de modestie}


Le Dictionnaire historique de la langue française (1998 F-PR : 2400) signale que nous (881), dont nos, terme latin, est à l'origine, est «quelquefois employé au sens d'ego « moi » $(\rightarrow$ je $)$, notamment pour exprimer l'opposition » :

Depuis le haut moyen âge (Léon le Grand), nos est employé comme pluriel dit de «majesté » lorsque le locuteur est une autorité religieuse et civile, et comme pluriel dit de «narration » pour représenter l'auteur qui s'exprime $\left(2^{\mathrm{me}}\right.$ moitié du VIII ${ }^{\mathrm{e}}$ s. $)$ ou bien l'auteur et ses lecteurs $\left(\mathrm{X}^{\mathrm{e}}\right.$ s. $)$. [...] En français, les emplois de nous comme pronom personnel sont fixés avant la fin du XII ${ }^{\mathrm{e}}$ s. [...] Des emplois stylistiques se sont développés au XIII ${ }^{\mathrm{e}}$ s. : nous désigne le locuteur seul, non associé à d'autres personnes (1200), spécialement dans l'emploi dit «de modestie » (1213) et comme en latin médiéval, dans celui dit «de majesté ${ }^{38}$, pour un grand personnage (1241). (Dictionnaire historique de la langue française 1998 F-PR : 2400)

Le pluriel de modestie renvoie donc à un emploi démarcatif - qui s'écarte de la norme (je) - pour désigner le locuteur aussi bien sur le plan syntaxique que référentiel. Or, cet emploi est indissociable d'un cadre énonciatif donné : il faut en effet qu'un certain nombre de propriétés sociolinguistiques soient réunies pour qu'on puisse y recourir. Dans le cas présent, il s'agit d'un cadre oral ou écrit à l'intérieur duquel un sujet unique s'exprime face à la communauté scientifique des " auteurs et conférenciers ${ }^{39}$ (Riegel et al. 2009 : 363). Concernant le mode écrit, il est précisé dans le Bon usage que «la $1^{\text {re }}$ pers. du pl. peut être employée au lieu du sg. dans le pluriel dit de modestie, quand un auteur parle de lui-même », ou encore «à l'impératif, parce que ce mode n'a pas de $1^{\text {re }}$ pers. du sg. » (Grevisse 1993 : 962). Or, cela ne va pas sans entraîner un marquage syntaxique particulier dans le reste de l'énoncé : "avec nous, qui est employé pour je seulement dans certains types d'écrits, il n'est pas rare de trouver des accords fautifs » (Grevisse 1993 : 962 ; c'est moi qui souligne) quant au nombre et au genre. Les conférenciers ou les auteurs n'ont en effet pas toujours conscience qu'un tel emploi est contraint sur le plan syntaxique en raison de sa signification référentielle unique. Par conséquent, l'accord avec le participe passé s'effectue au singulier et marque le genre naturel (masc. ou fém.), du moins dans la langue soutenue, ce qui relève d'une "syllepse obligatoire », comme l'indiquent les exemples du Bon usage (Grevisse 1993 : 659) : «Nous nous sommes résolu à publier les résultats de notre enquête (A. Martinet, Prononc. du fr. contemp., Introd.) »; «Nous nous sommes tenue aux éditions parues du vivant de Flaubert (Claudine Gothot-Mersch, dans Flaub., Mme Bov., p. 366) ».

La raison d'un tel emploi est liée à un procédé d'évitement de la $1^{\text {re }}$ personne du sg. je «qui apparaîtrait, comme moi, prétentieux» (Dubois et al. : 307). Ce qui va dans le sens de l'analyse de Benveniste selon lequel, «l'emploi de « nous » estompe l'affirmation trop tranchée de « je » dans une expression plus large et diffuse : c'est le

\footnotetext{
${ }^{38}$ Benveniste précise que dans ce cas, «le "je" s'amplifie par "nous" en une personne plus massive, plus solennelle et moins définie » Benveniste 1966: 235. C'est également la position de Riegel et al. 2009: 363 qui voient dans ce «substitut rhétorique de je» un emploi métaphorique du pluriel. Voir également Schmidely 1976: 71 et Maingueneau 1999: 33.

${ }^{39}$ Voir aussi Wagner, Pichon 1962: 167-168 cités par Kerbrat-Orecchioni 2009: 71. Hagège 1982: 97 parle, quant à lui, du «nous d'exposé savant».

Ce cadre énonciatif s'applique également à la communauté des apprenants, car, comme Dubois et al 1994: 307 l'indiquent, « le nous de modestie est en particulier une marque du discours didactique ». Voir également Maingueneau 1999: 32 à ce sujet.
} 
«nous » d'auteur ou d'orateur»(Benveniste 1966 : 235). Riegel et al. (2009: 363) estiment à la suite de ce linguiste que nous «estompe l'individualité derrière une identité collective ». Ainsi, pour résoudre le problème posé par le marquage de la subjectivité de la source énonciative, la langue dispose du «glissement d'une personne à une autre ", à savoir d'un «détournement de formes et non de formes réservées à cet effet » (Maingueneau 1999 : 27). Dans l'échelle des rangs personnels, je est inclus dans nous (Benveniste : 1966 : 233) et le sujet peut référer à lui-même sans employer la forme je de rang 1 en recourant au rang 4, à savoir nous. Je est perçu comme étant associé à la valeur contrastive de la forme disjointe moi, qui rompt la consensualité, en signifiant moi-je qui s'oppose à toi-tu, dans le champ de forces inter-sujets. Il s'agit là d'une interprétation argumentative de je, qui exploite la corrélation de subjectivité sur le mode sur-joué de l'altérité intersubjective : moi, pas toi. Là où je est donc considéré comme trop orgueilleux, ou risque d'être interprété comme tel, quand il est question d'exposer, voire de défendre, une thèse, il apparaît qu'il fait l'objet d'une valuation appréciative en bon/mauvais relative à une échelle argumentative (j'ai raison/tu as tort), ce qui explique son évitement par ceux qui ne veulent pas se situer ouvertement sur le terrain d'une controverse éventuelle. Culioli explique en effet que toute assertion est implicitement polémique :

Culioli : Je suis amené à définir l'assertion stricte : moi en tant que sujet, sujet c'est-à-dire source subjective...

NORMAND : source de mes paroles.

CULIOLI : source de mes paroles dans un champ intersubjectif, à l'intérieur d'une relation institutionnelle concernant un certain nombre de phénomènes, je dis - et en le disant je ne fais pas que le dire, ce ne sont pas que des mots, je m'engage en tant que sujet responsable de ce que je dis - je dis que je sais, pense, crois, que telle chose est le cas. Et en gros, ajouté, comme une sorte de coda, et si autrui veut me montrer que j'ai tort... !

NORMAND : qu'il parle ! (Culioli et Normand $2005: 166)$

Kerbrat-Orecchioni $(2009: 47 ; 71-72)$ voit dans cet emploi rhétorique un «trope syntaxique » (1986:107), et donc grammatical. Plus précisément, il s'agit, selon cette linguiste, d'une « énallage » de nombre/personne, qui consiste à employer une forme pour une autre « avec une valeur décalée par rapport à la valeur plus usuelle » (2009 : 70). Elle justifie d'ailleurs son propre choix stylistique de recourir au nous de modestie dans l'ouvrage cité (2009 : 71, note 1) mais aussi dans L'implicite de 1986 :

Dont nous faisons ici usage - sans en être autrement satisfaite du reste : ses connotations pompeuses et scolastiques, les problèmes d'accord qu'il soulève, tout cela nous gêne un peu aux entournures. Mais nous n'avons pu nous résoudre à employer systématiquement le «je », qui est encore, dans ce type de discours, trop «marqué » pour ne pas produire parfois certains effets indésirables, et dont le retour obstiné ne «passe » pas toujours très bien. (1986:362, note 12$)$

En raison de l'ancrage de cette structure linguistique dans un cadre énonciatif donné, défini par un genre de discours dont les spécificités ont été exposées ci-dessus, Kerbrat-Orecchioni considère que les énallages «investissent des unités déictiques » 
et relèvent ainsi de la «pragmatique énonciative », d'où leur appellation de «tropes pragmatiques » (1986: 107). Il convient alors de s'interroger sur les conditions de fonctionnement de ces derniers à travers les «indices» qu'ils laissent dans l'environnement co-textuel et contextuel de l'énoncé. En effet, pour fonctionner correctement, le trope doit être déchiffré, c'est-à-dire identifié pour ce qu'il est, à savoir l'emploi d'une forme plurielle pour une forme de singulier dans le cas du nous de modestie. Cette substitution est rendue manifeste sur le plan syntaxique (ou cotextuel) par l'accord du participe passé, comme évoqué précédemment, mais aussi par « la nature de certains prédicats, dont la spécificité invite à penser qu'ils ne peuvent raisonnablement s'appliquer qu'à une personne singulière »; par ailleurs, le contexte livre «des informations dont on dispose sur le sujet d'énonciation, directement »dans le cadre d'une conférence orale ou « indirectement » quand c'est « la signature » qui fonctionne «comme un indice cotextuel permettant la reconstitution du contexte énonciatif » (Kerbrat-Orecchioni 1986 : 146). L'exemple (9) permet d'illustrer ce fonctionnement syntaxico-pragmatique de l'énallage de nombre/personne :

(9) Grâce à elle [l'étude de Joly 1973] nous avons mieux compris les écrits de Moignet qui sont parfois, comme ceux de Guillaume, d'une lecture ardue - particulièrement l'article sur "Personne humaine et personne d'univers » (1970) - et nous avons été amené à nous interroger à nouveau sur le bienfondé des vues de Benveniste. (Schmidely 1976 : 53)

Le recours à nous permet à l'auteur de s'avancer à demi-masqué, pour ainsi dire, dans la mesure où il s'auto-désigne indirectement sur le plan linguistique. Nous est identifié au je de l'auteur notamment par l'accord en nombre (sg.) et en genre, qui manifeste ici l'opposition référentielle des sexes: en (9), l'accord au masculin sg. renvoie à un animé humain homme unique. Ce que corroborent les prédicats subjectifs «comprendre » et «être amené à s'interroger». La référence est identifiable comme telle également sur le plan extralinguistique, en relation avec le nom de l'auteur (prénom et nom de famille), indiqué en bibliographie : en (9) il s'agit de Jack Schmidely. Le référent unique de nous est donc situé non seulement par rapport à une coordonnée subjective, mais aussi par rapport à des coordonnées spatiotemporelles définies par le lieu et la date de la communication orale ou par le lieu d'édition et la date de publication de l'article écrit, en l'occurrence Montpellier et 1976 pour (9).

Ainsi, le pluriel est la trace d'un ajustement à une identité énonciative non fusionnelle, en tant que représentation symbolique de l'énonciateur-chercheur (ici, linguiste) dans un cadre discursif professionnel de type scientifique ${ }^{40}$.

\footnotetext{
${ }^{40}$ Voir Filippi-Deswelle 2012a pour de plus amples développements. Voir également l'analyse de Schmidely 1976: 71 en termes guillaumiens de mouvement d'interception en plusieurs points a, b, c et $\mathrm{d}$, parcourant les champs du «moi » et du «hors-moi »: «Au départ, en $a$, nous est le contenant de tous les locuteurs - et donc de tous les humains - possibles. Le mouvement engagé à partir de $a$ livre en discours un nous collectif et homogène, par lequel un ensemble, plus ou moins large, de locuteurs s'expriment à l'unisson. En $b$, la progression vers le hors-moi exige la définition du moi : il faut passer d'un locuteur collectif à un locuteur individuel. Si la saisie s'opère vers la fin de ce mouvement de rétrécissement - de "déplétion" ? - la pluralité livrée est restreinte par rapport à la position de départ, tout en étant orientée vers un moi unique non encore atteint : le moi transparaît sous le nous. C'est ainsi que nous décririons le nous de "modestie". En $c$, nous est intercepté au moment où il atteint la limite du champ du moi : le champ a été totalement parcouru, le seuil ouvrant à une pluralité réelle est atteint. Nous équivaut à moi tout en le «maximant» par l'idée de pluriel dont il est porteur. Le pluriel de «majesté » trouverait là son explication. En $d$, une fois franchi le champ du moi, nous livre une pluralité hétérogène qui, à partir de "moi + un autre" s'ouvre sur l'infinité des pluriels possibles. »
} 


\section{Conclusion}

Je conclurai cette présentation sur «les personnes grammaticales en relation » en disant que l'énonciation en première personne du pluriel n'est qu'un des protocoles énonciatifs possibles parmi les diverses combinaisons, en relation de complémentarité, qu'offre le système des personnes. En choisissant l'énonciation en première personne du singulier je, le chercheur ne fait pas tant preuve d'orgueil ou de manque de modestie qu'il ne s'expose à la confusion des rôles, des masques, des fonctions, et par là au brouillage de son identité. Mais il se trouve alors que, si l'auteur choisit de recourir à je, comme dans l'emploi du je performatif, le risque de confusion est heureusement levé par le contexte qui permet d'identifier l'énonciateur au chercheur uniquement : la distinction locuteur $\left(S_{1}\right)$ - énonciateur $\left(S_{0}\right) /$ support de la prédication $\left(\mathrm{S}_{2}\right)$ s'effectue alors seulement pragmatiquement, et non plus aussi morphologiquement comme avec nous.

\section{Bibliographie}

Arrive, Michel \& Gadet, Françoise \& Galmiche, Michel. 1986. La grammaire d'aujourd'hui, guide alphabétique de linguistique française. Paris: Flammarion.

Benveniste, Emile. 1966 [1946]. Problèmes de linguistique générale, Tome 1. Paris: Gallimard.

Benveniste, Emile. 1974 [1970]. Problèmes de linguistique générale, Tome 2. Paris: Gallimard.

Creissels, Denis. 1995. Eléments de syntaxe générale. Paris: P.U.F.

Culioli, Antoine. 1990. Pour une linguistique de l'énonciation, Opérations et représentations, Tome 1. Collection L'homme dans la langue animée par Janine Bouscaren. Paris: Ophrys.

Culioli, Antoine. 1999a. Pour une linguistique de l'énonciation, Formalisation et opérations de repérage, Tome 2. Collection L'homme dans la langue animée par Janine Bouscaren. Paris: Ophrys.

Culioli, Antoine. 1999b. Pour une linguistique de l'énonciation, Domaine notionnel, Tome 3. Collection L'homme dans la langue animée par Janine Bouscaren. Paris: Ophrys.

Culioli, Antoine. 2002. Variations sur la linguistique, Entretiens avec Frédéric Fau (préface et notes de Michel Viel). Paris: Klincksieck.

Culioli, Antoine \& Normand, Claudine. 2005. Onze rencontres sur le langage et les langues. Paris: Ophrys.

Damourette, Jacques \& Pichon, Edouard. 1927-1950. Essai de grammaire française. Des mots à la pensée, 7 volumes. Paris: d'Artrey.

Danon-Boileau, Laurent. 2007. Le sujet de l'énonciation, Psychanalyse et linguistique, (nouvelle édition augmentée). Paris: Ophrys.

Dictionnaire de la langue française. 1990. Paris: Le Petit Robert.

Rey, Alain (dir.). 1998. Dictionnaire historique de la langue française. Paris: Le Robert.

Dubois, Jean., Giacomo, Mathée., Guespin, Louis., Marcellesi, Christiane., Marcellesi, Jean-Baptiste et Mevel, Jean-Pierre. 1994 [2002]. Dictionnaire de linguistique. Paris: Larousse. 
Ducrot, Oswald. 1982. La notion de sujet parlant. Recherche sur la philosophie et le langage 2: 65-93.

Dufaye, Lionel et Khalifa, Jean-Charles. 2006. L'épreuve de grammaire à l'agrégation d'anglais. Paris, Ellipses.

Filippi-Deswelle, Catherine. Le sujet modal ou la construction du maître du je en question », Colloque international et interdisciplinaire La construction $d u$ sujet, organisé par Anne Besnault-Lévita et Natalie Depraz, à l'Université de Rouen, les 5-7 juin 2008, à paraître aux éditions Lambert-Lucas.

Filippi-Deswelle, Catherine. 2009. Anyway ou le mode énonciatif du savoir en prendre et en laisser. Anglophonia 26:121-150.

Filippi-Deswelle, Catherine. 2010. Quand «je» est un «autre» ou quand though, marqueur de relance discursive (d' «afterthought»), introduit une altérité intrasubjective. In L'Altérité dans les théories de l'énonciation, éd. Lionel Dufaye et Lucie Gournay, 37-55, Paris: Ophrys.

Filippi-Deswelle, Catherine. 2012a. Du locuteur au sujet énonciateur-locuteur, Théorisation des métatermes «locuteur/allocutaire » en «énonciateur/coénonciateur»: de la «mécanique interlocutoire » de Benveniste au couple énonciatif de Culioli. In Benveniste après un demisiècle, Regards sur l'énonciation aujourd'hui, éd. Lionel Dufaye et Lucie Gournay, 84-108, Paris: Ophrys.

Filippi-Deswelle, Catherine (éd.). 2012b. L'ajustement dans la TOE d'Antoine Culioli. In Epilogos, 3, Rouen: Publications Électroniques de l'ERIAC http://eriac.net/category/publications/publications-electroniques/epilogos/l-ajustementdans-la-toe-d-antoine-culioli/

Filippi-Deswelle, Catherine. 2012c. Quand l'adverbe connecteur anyway fraie le chemin de l'anaphore. In Anaphores et anaphoriques, Variété des langues, variété des emplois, éd. Camille Denizot et Emmanuel Dupraz, 343-362, Mont-Saint-Aignan: Publications des Universités et Rouen et du Havre.

Grevisse, Maurice. 1993. Le Bon usage, $13^{\mathrm{e}}$ édition. Bruxelles: De Boeck-Duculot.

Groussier, Marie-Line et Riviere, Claude. 1996. Les mots de la linguistique, Lexique de linguistique énonciative. Paris: Ophrys.

Hagege, Claude. 1982. La structure des langues. Paris: P.U.F.

Hagege, Claude. Benveniste et la linguistique de la parole. In E. Benveniste aujourd'hui, Actes du Colloque international du C.R.N.S., Université François Rabelais, Tours, 28-30 septembre 1983, Tome 1, éd. Guy Serbat, *****. Paris: Bibliothèque de 1'Information grammaticale.

Jacquesson, François. 2008. Les personnes, Morphosyntaxe et sémantique. Paris: C.N.R.S. Editions, 2008.

Jakobson, Roman. 1963. Essais de linguistique générale (tr. Nicolas Ruwet). Paris: Les Editions de Minuit.

Jespersen, Otto. 1922. Language, its nature, development and origin. Londres: Allen \& Unwin.

Joly, André. 1987. Sur le système de la personne. Revue des langues romanes, Tome LXXX, fasc. 1, Montpellier, 1973, p. 3-56. Repris dans André JOLY, Essais de systématique énonciative, Lille, P.U.L., 1987.

Joly, André. 1994. Eléments pour une théorie générale de la personne. Faits de langue 3: 45-54.

Kawagushi, Jungi. 1984. Le concept de personne. In E. Benveniste aujourd'hui, Actes du Colloque international du C.R.N.S., Université François Rabelais, Tours, 
28-30 septembre 1983, Tome 1, éd. Guy Serbat, *****. Paris: Bibliothèque de l'Information grammaticale.

Kerbrat-Orecchioni, Catherine. 1986. L'implicite. Paris: Armand Colin.

Kerbrat-Orecchioni, Catherine. 2009. L'énonciation. Paris: Armand Colin.

Lallot, Jean. 1998. La grammaire de Denys le Thrace (tr. Jean Lallot). Paris: C.N.R.S. Editions.

Letoublon, Françoise. 1994. La personne et ses masques. Faits de langue 3: 7-14.

Lyons, John. 1984. La subjectivité dans le langage et dans les langues. In $E$. Benveniste aujourd'hui, Actes du Colloque international du C.R.N.S., Université François Rabelais, Tours, 28-30 septembre 1983, Tome 1, éd. Guy Serbat, $*_{*} * *$. Paris: Bibliothèque de l'Information grammaticale.

Maillard, Michel. 1994. La catégorie de la personne fait-elle partie des universaux du langage ?. Faits de langue 3: 53-62.

Maingueneau, Dominique. 1990. Eléments de linguistique pour le texte littéraire, Paris: Bordas.

Maingueneau, Dominique. 1999. L'énonciation en linguistique française. Paris: Hachette Supérieur.

Meillet, Antoine. 1937. Introduction à l'étude comparative des langues indoeuropéennes, $8^{\mathrm{e}}$ édition. Paris: Hachette.

Moignet, Gérard. 1972. Sur le système de la personne en français. Travaux de linguistique et de littérature, Centre de Philologie et de Littératures romanes de l'Université de Strasbourg.

Riegel, Martin., Pellat, Jean-Christophe et Rioul, René. 2009. Grammaire méthodique $d u$ français, Edition revue et augmentée. Paris: P.U.F.

Schmidely, Jack. 1976. Controverse à propos du système de la personne. Revue des langues romanes, Tome LXXXII: 53-84.

Vogue, Sarah (de). 1992. Culioli après Benveniste : énonciation, langage, intégration. Revue LINX 26: 77-108.

Wagner, Robert-Léon et Pinchon, Jacqueline. 1962. Grammaire française classique et moderne. Paris: Hachette.

Wilmet, Marc. 2003. Grammaire critique $d u$ français, $3^{\text {ème }}$ édition. Bruxelles: Duculot. 